Portland State University

PDXScholar

Winter 3-28-2019

\title{
Investigation of Electronic and Optical Properties of 2-Dimensional Semiconductor Tin Selenide (SnSe) Thin Films
}

Shakila Afrin

Portland State University

Follow this and additional works at: https://pdxscholar.library.pdx.edu/open_access_etds

Part of the Electrical and Computer Engineering Commons Let us know how access to this document benefits you.

\section{Recommended Citation}

Afrin, Shakila, "Investigation of Electronic and Optical Properties of 2-Dimensional Semiconductor Tin Selenide (SnSe) Thin Films" (2019). Dissertations and Theses. Paper 4862.

https://doi.org/10.15760/etd.6738

This Thesis is brought to you for free and open access. It has been accepted for inclusion in Dissertations and Theses by an authorized administrator of PDXScholar. Please contact us if we can make this document more accessible: pdxscholar@pdx.edu. 
Investigation of Electronic and Optical Properties of 2-Dimensional Semiconductor Tin Selenide (SnSe) Thin Films

by

Shakila Afrin

A thesis submitted in partial fulfillment of the requirements for the degree of

\author{
Master of Science \\ in \\ Electrical \& Computer Engineering
}

Thesis Committee:

Raj Solanki, Chair

Richard Campbell

Shankar Rananavare

Portland State University

2019 
(C)2018 Shakila Afrin 


\begin{abstract}
Over the last 5 decades, the semiconductor industry has been well served by Si based technology due to its abundant availability, lower manufacturing cost, large wafer sizes and less complexity in fabrication. Over this period, electronic devices and integrated systems have been miniaturized by downscaling of the transistors. The miniaturization has been guided by the Moore's law where the numbers of transistors have doubled over every two years. However, the trend of transistor miniaturization is fast approaching its limit. Hence, alternate and innovative solutions are necessary to tackle this problem and this propels the research for finding novel materials with unique properties.

The isolation of graphene, a single layer of graphite in 2004 had dramatically pioneered a new regime of research and investigation as a potential material to replace traditional Si. Graphene is the most widely studied two dimensional (2D) material exhibiting fascinating electronic, optoelectronic and electrochemical properties. Room temperature graphene has very high carrier mobility, a hundred times larger than that of $\mathrm{Si}$, but it lacks a bandgap preventing its application in digital electronics. However, the advent of graphene initiated exploration of other 2D materials as a possible replacement for Si for future generation of electronic devices. Other 2D layered materials include transition metal dichalcogenides (TMDs), other layered metal chalcogenides, black phosphorus (BP), boron nitride $(\mathrm{BN})$ etc which are also attractive due to fascinating electronic band structure and layer dependent properties that have demonstrated potential applications in optoelectronics and semiconductor devices. Metal chalcogenides are among the wellstudied layered materials that have been isolated as high-quality and two-dimensional crystals. Among the 2D layered metal chalcogenide materials is tin selenide ( $\mathrm{SnSe}$ ), which


belongs to group IV-VI that has attracted considerable attention due to its interesting structural and optical properties, hence it has potential applications in optoelectronics, photovoltaics, memory, energy storage, and catalysis.

To date, SnSe films have been produced by exfoliation or chemical vapor deposition that produces flaky films. In this research, uniform, smooth and high quality SnSe thin films were grown over large area $(5 \mathrm{~cm} \times 5 \mathrm{~cm}) \mathrm{Si} / \mathrm{SiO}_{2}$ substrates using Atomic Layer Deposition (ALD). Films were grown over a temperature range of $350^{\circ} \mathrm{C}$ to $450^{\circ} \mathrm{C}$, which exhibit p- type semiconductor characteristics. ALD is perfect for the growth of layered materials due to its precise controllability of film composition and thickness as the growth proceeds layer by layer. Structural and optical properties of the as-grown films were investigated using X-ray diffraction (XRD), Raman spectroscopy, and X-ray photoelectron spectroscopy (XPS). These analyses show growth of 2 dimensional, orthorhombic phase films. Magnetic analysis shows a paramagnetic behavior. Back-gated transistors were fabricated for electrical characterization which showed p-type conductance, with an average hole mobility of $10 \mathrm{~cm}^{2} / \mathrm{V}$.s and $\mathrm{I}_{\mathrm{on}} / \mathrm{I}_{\text {off }}$ ratio of $\sim 10^{5}$. 
Dedication

To my parents 


\section{Acknowledgments}

I would like to acknowledge my supervisor Prof. Raj Solanki for supporting me in this research. Dr. Solanki has been a continuous source of knowledge and encouragement throughout my research, without his guidance I would not be able to succeed in this endeavor.

I owe my gratitude to all of the defense committee members, Prof. Richard Campbell and Prof. Shankar Rananavare for their kind advice and suggestion in general.

I would like to thank Capri Price from Dr. Clare's lab for assisting me in Raman analysis. I also would like to express my gratitude to Sheng Kuei Chiu from Dr. Goforth's lab for helping with the XRD measurements.

I would also like to thank ECE Chair Prof. Douglas Hall for his academic advice. His guidance helped me to set my career goal.

I thank to my colleagues Neal Kuperman and Michael Hopkins for providing help in the Lab of Naonoelectronics. Special thanks to Robert Browning for helping me with the fabrication. 


\section{Table of Contents}

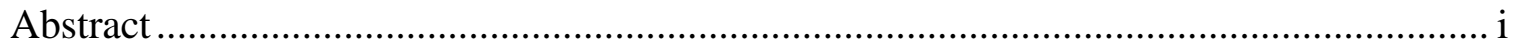

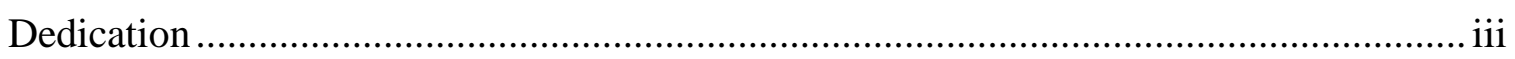

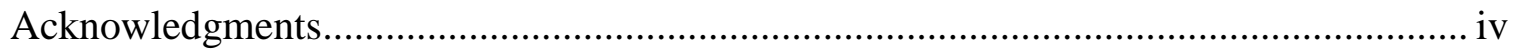

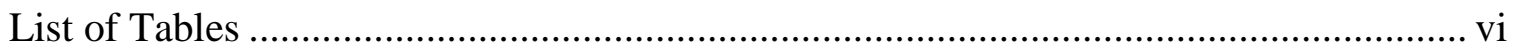

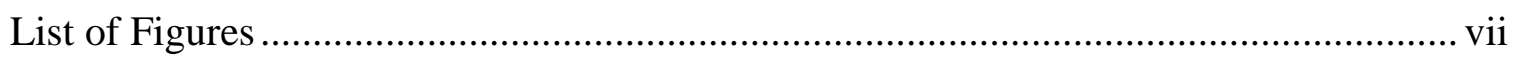

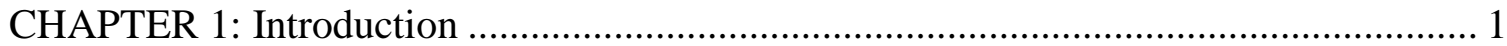

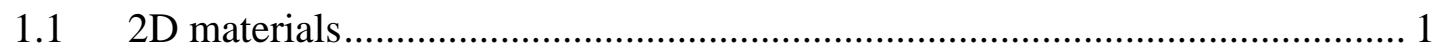

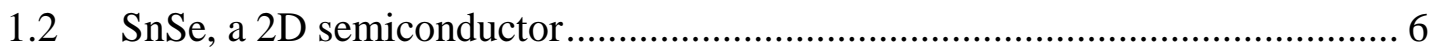

1.3 Contribution of this research ............................................................... 10

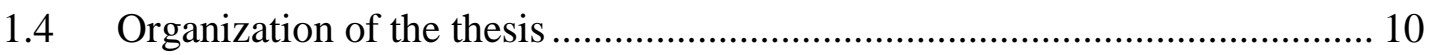

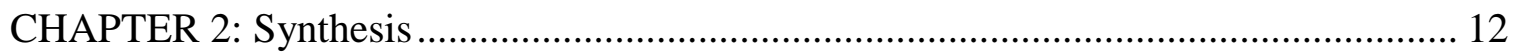

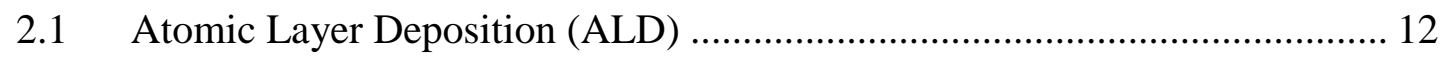

2.2 Synthesis of Tin Selenide (SnSe) using ALD .......................................... 14

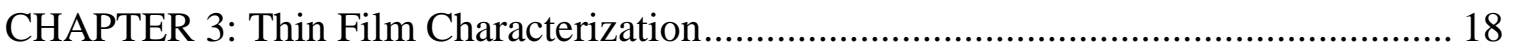

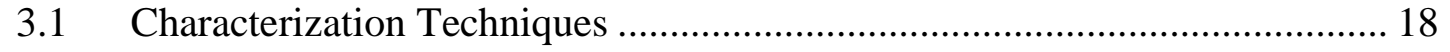

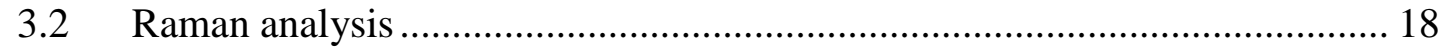

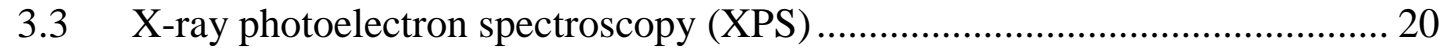

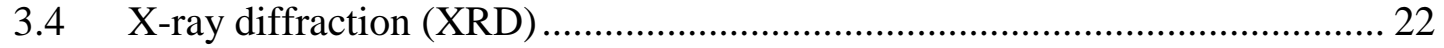

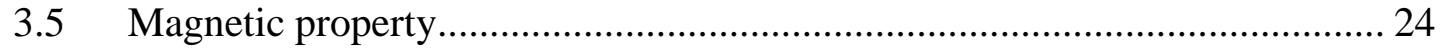

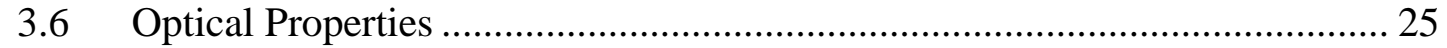

CHAPTER 4: Device fabrication and electrical characterization................................ 28

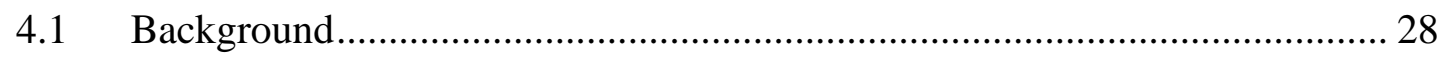

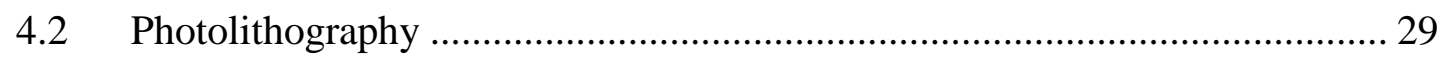

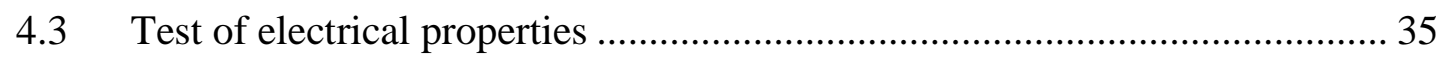

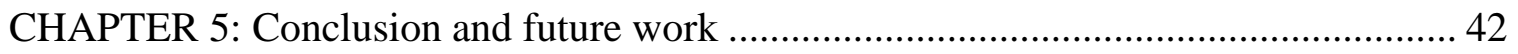

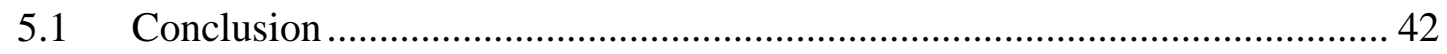

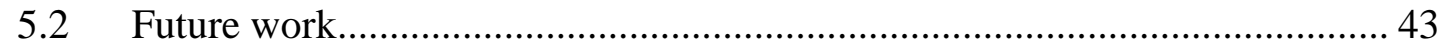

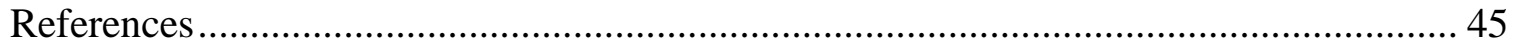




\section{List of Tables}

Table 1: Optical bandgap energies of different shaped SnSe materials in eV [60]......... 25

Table 2: Literature review of SnSn hole mobility reported by various techniques......... 40 


\section{List of Figures}

Figure 1. 1: Elements comprising most of 2D materials [1] ........................................ 1

Figure 1. 2: Library of some of more common 2D layered materials [4]....................... 2

Figure 1. 3: Crystal structures and interlayer distance of three important 2D layered

materials graphene, molybdenum disulphide and tin selenide are shown in a, b and c respectively [5-7].

Figure 1. 4: 2D layered materials with a broad spectral range (a) electromagnetic (EM) spectrum represented by a rainbow arrow. Top portion of the panel illustrates potential applications for different spectral ranges. Bottom panel represents the atomic structures for some of the key 2D layered materials: hexagonal boron nitride $(\mathrm{hBN})$, molybdenum disulfide ( $\mathrm{MoS}_{2}$ ), black phosphorus (BP), and graphene (from left to right). The possible spectral ranges covered by different materials are indicated using colored polygons. Electronic band structures of single layer $\mathrm{hBN}, \mathrm{MoS}_{2}, \mathrm{BP}$, and graphene are shown in (b), (c), (d) and (e) respectively [8]

Figure 1. 5: SnSe crystal structure along $b$ axis at room temperature. ............................. 8

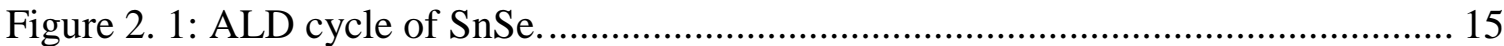

Figure 2.2: Microchemistry F-120 ALD reactor......................................................... 16

Figure 3. 1: Raman spectrum of as-grown $\mathrm{SnSe}$ thin film on $\mathrm{Si} / \mathrm{SiO}_{2}$ substrate [52]....... 19

Figure 3. 2: VersaProbe II.................................................................................. 20

Figure 3. 3: X-ray photoelectron spectroscopy (XPS) on SnSe thin film (a) shows Se 3d

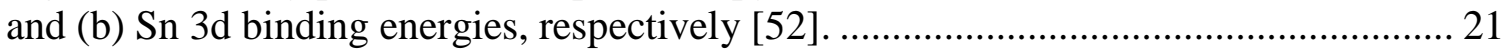

Figure 3. 4: X-Ray diffraction pattern of SnSe thin film grown by ALD [52]................ 23

Figure 3. 5: Magnetic properties of thin film SnSe illustrating a paramagnetic profile

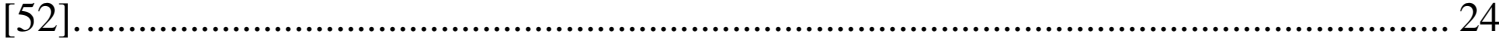

Figure 3. 6: Optical band structures of a) single-layer, b) double-layer, and c) bulk SnSe.

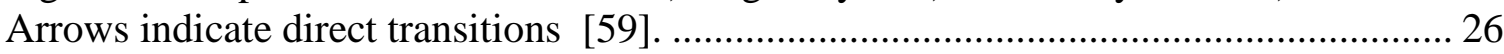

Figure 3. 7: Energy band gap calculation of ALD synthesized SnSe thin film............... 27

Figure 4. 1: Schematic of ALD synthesized $\mathrm{SnSe}$ thin film on $\mathrm{Si} / \mathrm{SiO}_{2}$ substrate............ 29 Figure 4. 2: Contact Mask for patterning.................................................................... 30 Figure 4. 3: Spin coater P6700 used to coat the wafer using photoresist. ......................... 31 Figure 4. 4: OAI Hybralign 200 contact Aligner used for Lithography exposure............. 32 Figure 4. 5: Optical Microscope used for imaging at different steps of the fabrication. .. 33 Figure 4. 6: a) Schematic diagram of a SnSe back-gated transistor, b) patterned features on the substrate after metallization showing array of devices, c) optical view of the patterned substrate focusing on a cluster of transistors of gate lengths of 2, 4, 6, 8, and 10

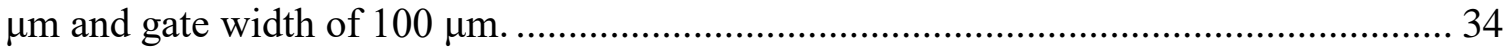

Figure 4. 7: Probe Station used for electrical testing...................................................... 35

Figure 4. 8: HP 4145B Semiconductor Parameter Analyzer. .......................................... 36

Figure 4. 9: Electrical probing of the back gated SnSe transistors................................... 36 
Figure 4. 10: Linear $I_{d}-V_{d}$ characteristic of SnSe thin film with Au electrodes at zero gate

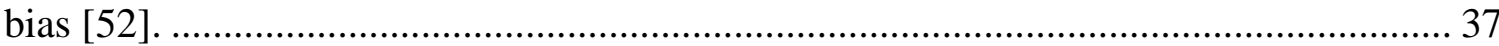
Figure 4. 11: Current-voltage characteristics of a SnSe FET under illumination at zero

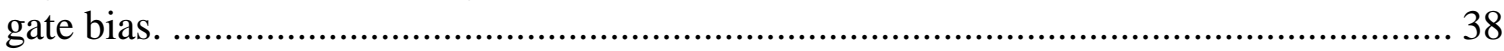

Figure 4. 12: Transfer characteristics of a SnSe p-FET [52] ........................................... 38

Figure 4. 13: Transfer characteristics of a SnSe n-FET.................................................. 39

Figure 5 1: Schematic diagram of a Top-gated SnSe FET transistor. .............................. 44 


\section{CHAPTER 1 \\ Introduction}

\subsection{D materials}

Significant research effort has been directed towards the search for new materials with unique properties that can shape the technological advancement of electronic devices. Graphene is one such new material discovered lately and has true potential to be used for advanced technological applications due to its novel properties. It was first isolated in 2004 from its parent material graphite in the form of a single crystal mono layer. The invention of graphene showed that the two-dimensional (2D) material properties can be significantly different and far superior to those of the bulk materials. The term "two-dimensional (2D)" implies that the material thickness is much smaller than the wavelength of the light. Graphene soon became the role model and the most studied 2D material due to its exceptional electronic, optoelectronic, and sensing applications. Although graphene has many unique properties, it has its own limitations hindering practical applications.

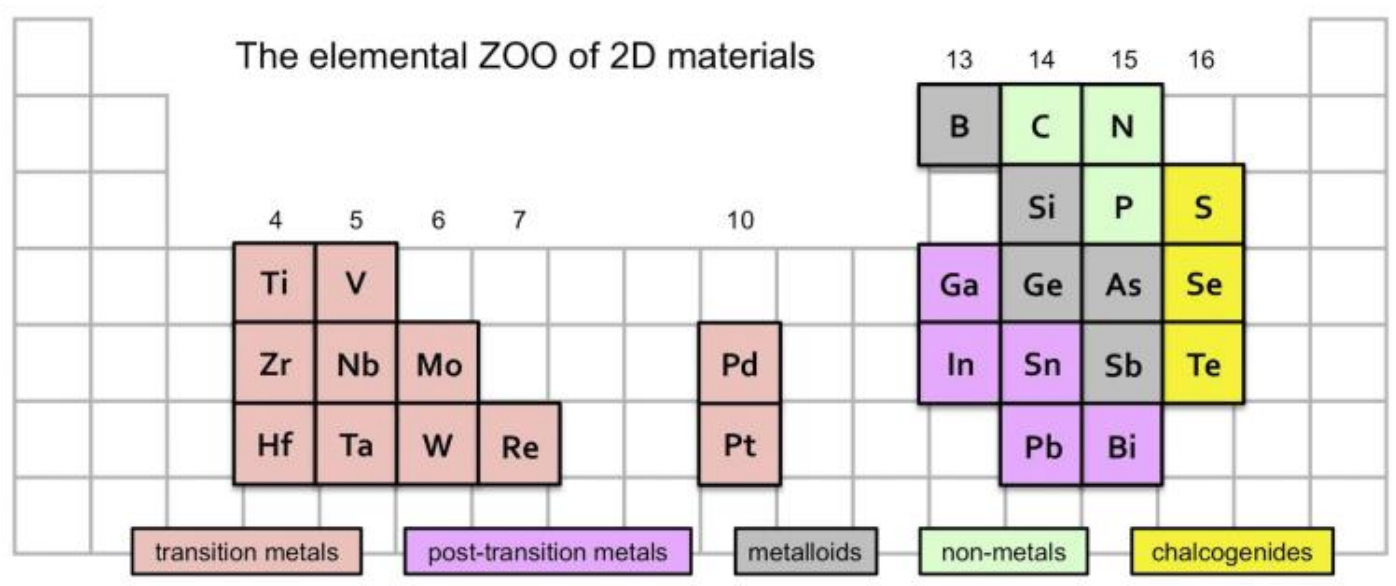

Figure 1. 1: Elements comprising most of 2D materials [1]. 
The lack of an intrinsic band gap is one of the major challenges restricting its potential to be fully utilized in electronics. However, graphene's discovery sparked off tremendous interest toward other alternate 2D semiconductors.

Following the success of graphene, researchers started to explore other two dimensional (2D) layered materials with an aim to addressing the desired properties for the next generation of electronic devices. Hence, a large number of 2D materials with various properties were investigated in the form of isolated monolayers. Fig. 1.1 shows the most common elements in the periodic table that comprise $2 \mathrm{D}$ materials. The most common layered 2D materials include hexagonal boron nitride $(\mathrm{hBN})$, transition metal, non-metal chalcogenides, oxides, hydroxides and halides. Hexagonal 2D boron nitride $(\mathrm{hBN})$ attracts a lot of research interest due to its potential to inducing an energy bandgap in graphene when graphene was exfoliated onto it [2].

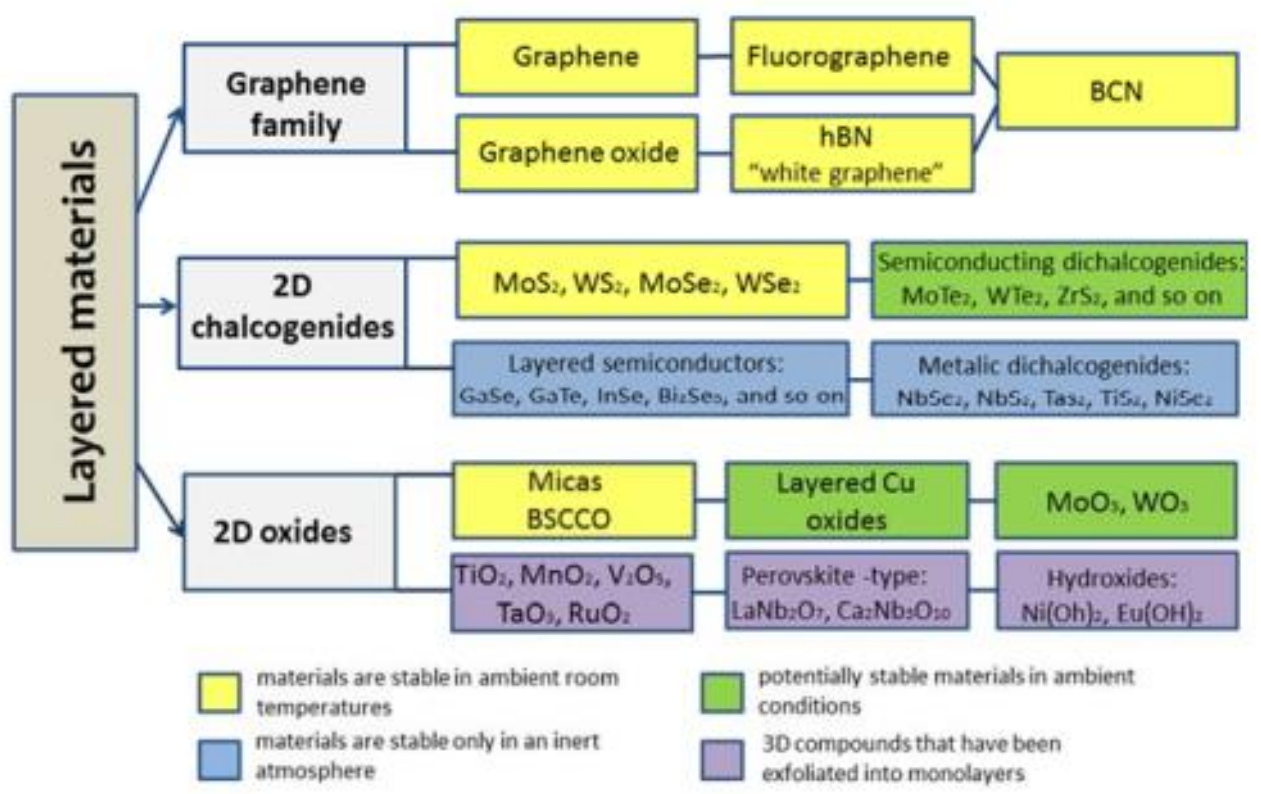

Figure 1. 2: Library of some of more common 2D layered materials [4]. 
Transition metal dichalcogenides (TMDCs) such as $\mathrm{MoS}_{2}, \mathrm{MoSe}_{2}, \mathrm{WS}_{2}$, $\mathrm{WSe}_{2}$, etc. are among the highly studied layered 2D materials due to their novel properties. Outside TMDCs, transition metal chalcogenides $\left(\mathrm{NbSe}_{3}, \mathrm{TaSe}_{3}\right)$ and transition metal oxides (TMOs: $\mathrm{LaVO}_{3}, \mathrm{LaMnO}_{3}$ ) are also isolated in monolayer form and are well studied [3]. Metal chalcogenides have also received particular attention, owing to their fascinating electronic band structures and remarkable layer-dependent properties. Fig. 1.2 illustrates a partial list of the most common 2D layered materials and some of their properties.

(a)

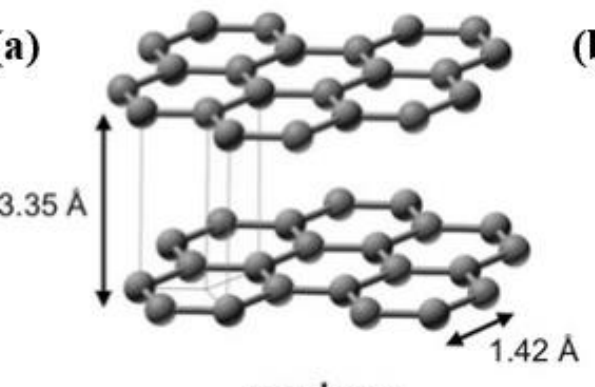

graphene (b)

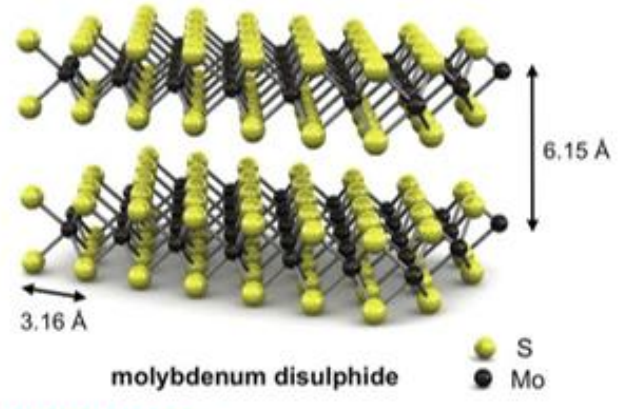

(c)
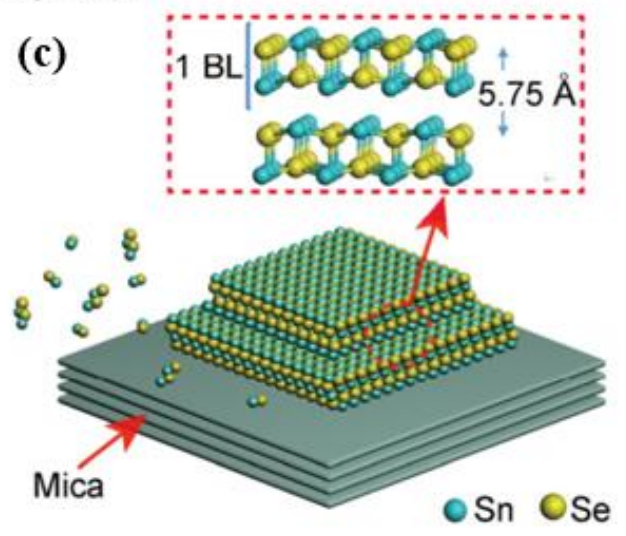

Figure 1. 3: Crystal structures and interlayer distance of three important 2D layered materials graphene, molybdenum disulphide and tin selenide are shown in a, b and c respectively [5-7].

Crystal structures of graphene, $\mathrm{MoS}_{2}$ (TMDC) and SnSe (Metal Chalcogenide) bilayers are illustrated in Fig. 1.3. In 2D layered materials, the interlayers are connected through weak van der Walls interaction while within the same layer the atoms are 
covalently bonded. Inter layer distances for some of the key 2D layered materials are noted in the Fig. $1.3(\mathrm{a}-\mathrm{c})$.

Unlike typical nanostructures, the surface of 2D layered materials are naturally passivated due to their dangling-bond-free atomic structures. This superior dangling-bondfree lattice structure makes them easily exfoliated or synthesized and integrated with various electronic and photonic devices at the atomic scale. Heterogeneous integration is another key advantages of 2D layered materials and allows significant freedom in fabricating complex heterostructures using various nanoscale and other 2D materials. 2D layered materials do not have the constraints of crystal lattice mismatch since layers with different lattice constants are bonded by weak van der Waals force. Thus they can be exploited to create novel devices with van der Waals heterostructures (vdWHs). Due to their diverse electronic and optoelectronic properties, 2D materials provide a wide range of Electromagnetic (EM) spectrum from radio wave to ultraviolet as seen in Fig. 1.4. Graphene has zero bandgap and it interacts with light from microwave to ultraviolet spectrum thus making it ideal candidate for signal modulation, light detection, etc. Its gapless nature hinders application in the light emitting devices. $\mathrm{MoS}_{2}$, one of the promising transition metal dichalcogenides (TMDCs), attracts its potential for light emitting properties primarily in near-infrared wavelength range due to its direct bandgap of $\sim 1.84$ $\mathrm{eV}$. Hexagonal boron nitride $(\mathrm{hBN})$ is another important type of 2D material, it has a large bandgap of around $6 \mathrm{eV}$, making it an excellent dielectric. Hexagonal boron nitride (hBN) is typically integrated in various heterostructures for electrostatic gating of other $2 \mathrm{D}$ materials [2]. Bulk black phosphorus (BP) has a direct bandgap of around $0.3 \mathrm{eV}$, which 
can be increased as high as $\sim 2 \mathrm{eV}$ in monolayer form, is an excellent material for nearinfrared to mid-infrared wavelength applications.

a
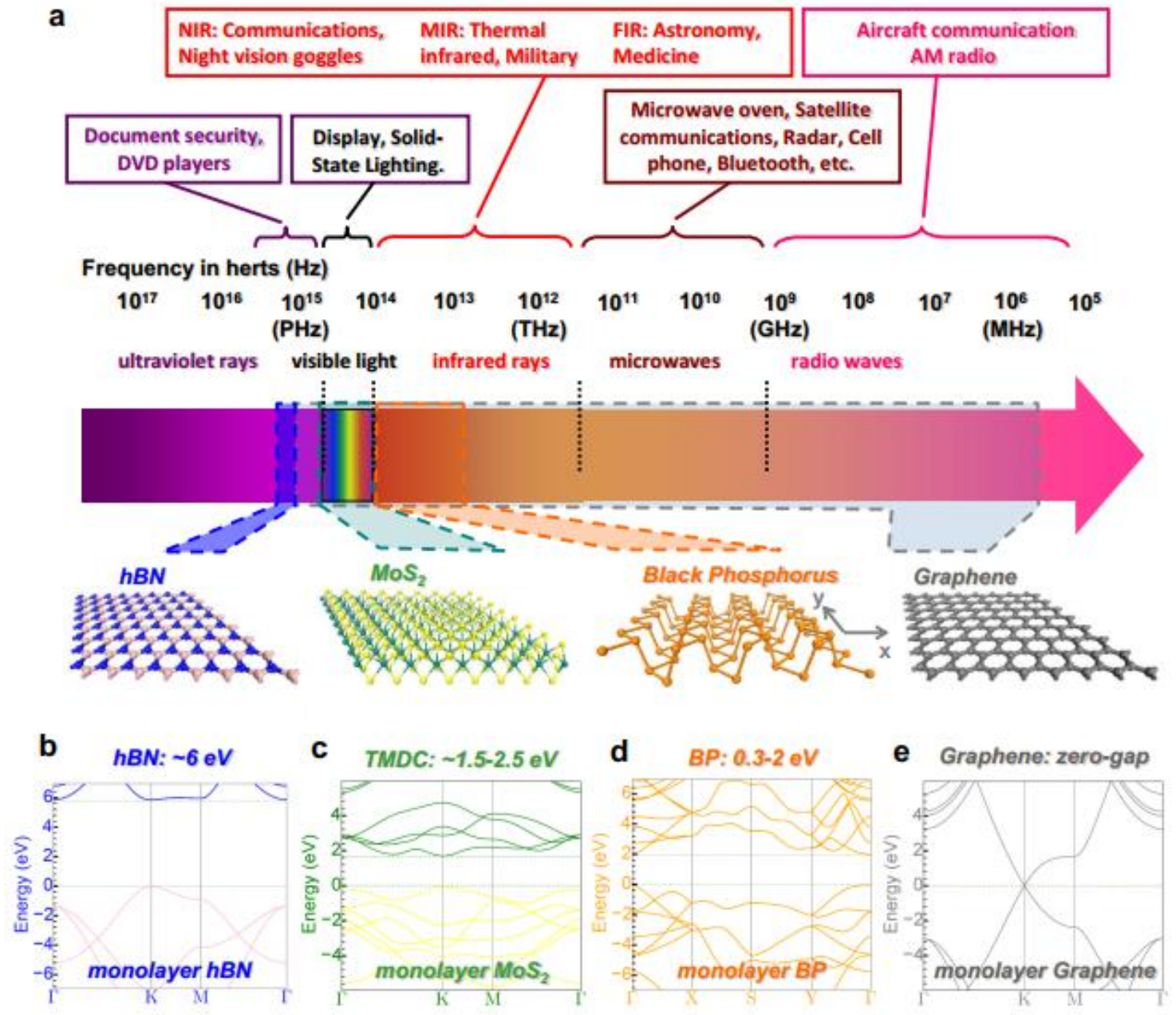

Figure 1. 4: 2D layered materials with a broad spectral range (a) electromagnetic (EM) spectrum represented by a rainbow arrow. Top portion of the panel illustrates potential applications for different spectral ranges. Bottom panel represents the atomic structures for some of the key 2D layered materials: hexagonal boron nitride (hBN), molybdenum disulfide $\left(\mathrm{MoS}_{2}\right)$, black phosphorus (BP), and graphene (from left to right). The possible spectral ranges covered by different materials are indicated using colored polygons. Electronic band structures of single layer $\mathbf{h B N}, \mathrm{MoS}_{2}, \mathrm{BP}$, and graphene are shown in (b), (c), (d) and (e) respectively [8]. 


\subsection{SnSe, a 2D semiconductor}

Extensive research has been conducted on 2D layered metal chalcogenides due to their unique surface and layer- dependent properties [9-10]. Metal chalcogenides have a very wide range of bandgaps which offer multiple applications in electronic, optical, and optoelectronic fields. Among the various metal chalcogenides, Tin-based binary chalcogenide compounds Sn-X (where X denotes a chalcogen: S, Se, Te) are particularly interesting due to their potential applications in the next generation devices and have been heavily investigated. Hence, the focus of this research was to examine the electrical, optical and the crystal structure of SnSe.

The layered 2D Sn-X compounds are typically crystallized into three phases: hexagonal, monoclinic, and orthorhombic. Chemical formula $\mathrm{Sn}-\mathrm{X}_{2}$ represents the hexagonal and monoclinic phases, while orthorhombic crystal structure is denoted by Sn$\mathrm{X}$. Sn-X materials are less researched compared to the $\mathrm{Sn}-\mathrm{X}_{2}$ family despite the fact that they are the ideal candidate materials for photovoltaic and optoelectronic applications with suitable energy band structures and excellent electronic properties [11-12]. However, the recent discovery of tin selenide $(\mathrm{SnSe})$ as a superior thermoelectric (TE) material has refocused the research interest on $\mathrm{Sn}-\mathrm{X}$ compounds.

Thermoelectric (TE) materials have been investigated extensively for energy harvesting and possible thermal power management applications such as in refrigerator or in heat pump. These types of materials have the ability to provide direct conversion between electric and thermal energy and the energy conversion efficiency is primarily represented by material dimensionless term called figure of merit (ZT). ZT defines how efficient a material can provide interconversion between thermal energy and electrical 
energy (or vice versa). Traditionally, group IV-VI compounds and their alloys such as $\mathrm{PbTe}, \mathrm{PbSe}, \mathrm{GeTe}$ and $\mathrm{SnTe}$ are well recognized as efficient thermoelectric materials owning to their unique structural properties [13-16]. Recent investigations confirmed that group IV-VI tin-based mono-chalcogenides $(\mathrm{Sn}-\mathrm{X})$ such as $\mathrm{SnSe}$ and $\mathrm{SnS}$ also hold a lot of promise for their potential in thermoelectric applications. Recent studies by Zhao et al. shows a high ZT of $2.6+/-0.3$ at $923 \mathrm{~K}$ in single crystal $2 \mathrm{D} \mathrm{SnSe,} \mathrm{which} \mathrm{is} \mathrm{far} \mathrm{superior}$ compared to the other popular TE materials [17]. At room temperature, SnSe belongs to Pnma space group with orthorhombic structure, however it converts to a high symmetry orthorhombic $\mathrm{Cmcm}$ space group at high temperature around $800 \mathrm{~K}$ [17-18]. Fig. 1.5 shows the room temperature lattice structure (Pnma group) of layered SnSe with orthorhombic crystal structure. For each of the layers, Sn and Se (S) atoms are bonded covalently and fabricate zig-zag formats along the $b$-axis [Fig. 1.5]. Interlayers are bonded by the weak van der Walls forces. The interlayer chalcogen to chalcogen distance in a unit cell is around $0.34 \mathrm{~nm} .[19]$.

The high $Z T$ value of $2 \mathrm{D}$ SnSe materials is explained by the compounding effects of its ultra-low intrinsic thermal conductivity $\left(<0.4 \mathrm{Wm}^{-1} \mathrm{~K}^{-1}\right.$ at $923 \mathrm{~K}$ along the $b$-axis $)$ and low power factor $\left(<10 \mathrm{uW} \mathrm{cm}{ }^{-1} \mathrm{~K}^{-2}\right.$ at $\left.900 \mathrm{~K}\right)$. Immense research effort has been focused on SnSe to increase the energy conversion efficiency (ZT) value by tuning hole-doping. [20]. 


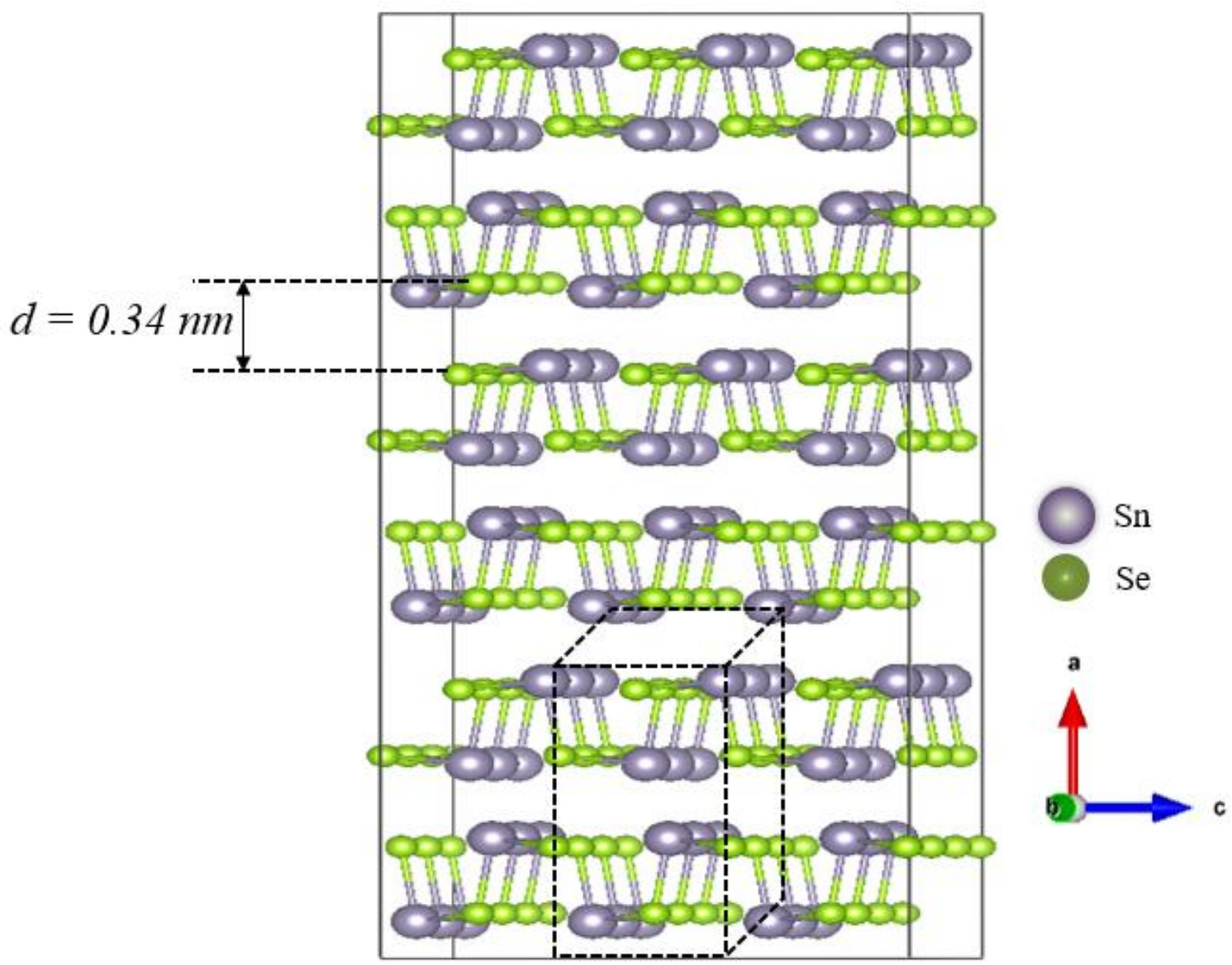

Figure 1. 5: SnSe crystal structure along b axis at room temperature.

Tin selenide ( $\mathrm{SnSe}$ ) belongs to IV-VI group which has attracted great attention due to its interesting structural and optical properties, hence has potential applications in optoelectronics, photovoltaics, memory, energy storage, and catalysis [12, 21-25]. The crystal structure of $\mathrm{SnSe}$ is orthorhombic with a direct band gap of about $1.3 \mathrm{eV}$, well suited for applications in photovoltaics and optoelectronics devices. Single crystal SnSe, which exhibits p-type semiconductor property, has potential applications in memory switching, owing to its interesting polarity dependent electronic properties [26]. Both tin and selenium, the constituents of SnSe are nature-abundant, as well as non-toxic, which makes 
it very suitable for the consumer market. 2D $\mathrm{SnSe}$ thin films display superior photosensitivity and tunable bandgap over bulk form due to higher surface area and quantum confinement effects.

SnSe has become more and more popular recently, yet there is a need to find feasible and controllable method to isolate or synthesize 2D single-crystal. The single and few layers SnSe thin films can be made by top-down mechanical exfoliation or bottom-up chemical synthesis process. Mechanical exfoliation has been used to prepare monolayer or few layer graphene, $\mathrm{MoS}_{2}$ and black phosphorus (BP) due to its low cost and high quality output. However a recent study by Hong-Yue et al. suggests that it might be difficult to mechanically exfoliate monolayer $\mathrm{SnSe}$ from its bulk phase due to the stronger inter-layer interaction [27]. It has been shown that the inter-layer binding energy (BE) of SnSe is much larger than that of graphite, $\mathrm{MoS}_{2}$ and BP. Based on density functional theory (DFT) the strength of inter-layer binding (meV/atom) for $\mathrm{SnSe}$ shows same trend using different exchange-correlation functionals, hence mechanical exfoliation might not be ideal technique for isolating SnSe from bulk phase.

In the literature a number of bottom-up techniques have been reported for synthesis of tin selenide ( $\mathrm{SnSe})$, including aqueous solution [27], thermal evaporation [28], electrodeposition [30], spray pyrolysis [31-33], hot wall epitaxy [34], vacuum evaporation [35], laser ablation [36], D.C. magnetron sputtering [37], chemical vapor deposition [38], and chemical bath deposition [39]. The films produced by these techniques are rough and composed of particulates. Unlike these techniques, we have employed atomic layer deposition (ALD) for growth of smooth, continuous films of SnSe. 
ALD is a step-wise growth process where reactants of the material are alternately injected into the growth area, followed by purging of the excess species and by-products after each growth cycle. The sequential surface reactions produce high quality films and could be grown over large substrates [40]. ALD is perfect for the growth of layered materials due to its precise controllability of film composition and thickness as the growth proceeds layer by layer. We have used this method to produce several layers thick, smooth and continuous thin films of $\mathrm{SnSe}$ over large areas $(5 \times 5 \mathrm{~cm})$. The films have been characterized by various techniques to determine their physical and electronic properties.

\subsection{Contribution of this research}

To date, the SnSe films produced suffer from size limitation (exfoliation) or grainy texture for films grown via various chemical vapor deposition (CVD) or physical vapor deposition (PVD) or other growth processes. Because of these limitations, ALD was investigated for growth of SnSe films. It is shown that using ALD, smooth and continuous films can be grown over large areas. To evaluate the quality of these films, their structural, optical and electrical properties have been characterized. It is shown that the quality of these films match those of exfoliated films, but are of much larger areas.

\subsection{Organization of the thesis}

The thesis starts (Chapter 1) with an introduction to two dimensional (2D) layered materials. The isolation of graphene from graphite and the birth of $2 \mathrm{D}$ materials are presented at first. Recent advancements in "beyond graphene" 2D materials is highlighted 
next. The importance of IV-VI metal chalcogenide tin selenide ( $\mathrm{SnSe}$ ) for next-generation of devices are also emphasized in this chapter. Chapter 2 exclusively focuses on the synthesis of tin selenide ( $\mathrm{SnSe}$ ) thin films. A comprehensive study of Atomic Layer deposition technique including benefits and growth mechanism is discussed in this chapter. Chapter 3 deals with different structural characterization techniques of the as-grown $\mathrm{SnSe}$ thin films. The development of the SnSe based back gated field effect transistor is described in Chapter 4. The details of the fabrication steps are presented here along with the electrical testing. Chapter 5 summarizes the major contributions of this work. Also we propose future extension of this work and further development. 


\section{CHAPTER 2 \\ Synthesis}

\subsection{Atomic Layer Deposition (ALD)}

There has been a continuous push to develop new materials with different dimensionality and functionality for applications in various fields such as electronics, photonics, energy harvesting and sensing. Atomically thin nanostructured materials serve the building blocks of highly promising advanced devices due to their superior and unique properties with down-scaling of device dimensions. In order to continue the relentless miniaturization, the atomically thin materials need to be coupled with different materials with precise controllability. Modern advanced devices use complex heterostructures to take advantage of the surface modification and fabrication steps. However, the synthesis of heterostructure materials on a limited space and high aspect ratio is very challenging. Furthermore, the synthesis of novel heterostructures based nanomaterials where one material needs to be grown on top of others with substantial surface area and chemical, physical resistivity is a major challenge. This requires a synthesis technique that is capable of providing atomic level procession while maintaining the native characteristics intact. Among all the various available deposition techniques, atomic layer deposition (ALD) appears to be one of the most promising techniques due to its simplicity, reproducibility and the high conformality of as-grown films. ALD has already been in widespread use in microelectronic and semiconductor industries because of its compatibility with modern micro/nano-fabrication processes. Modern CMOS fabrication processes use ALD to deposit gate dielectrics to produce conformal and homogenous thin films [41]. ALD is used as the primary deposition 
technique for high aspect ratio structures, various nanostructures such as nanoparticles, nanowires, nanotubes and biological materials [42-45].

Atomic layer deposition (ALD) is a step-wise growth technique where reactants or precursors of the material are alternatively injected into the growth area, followed by purging of the excess species and by-products after each growth cycle. The principle of the ALD technique is basically a reaction between the precursor materials where they are separated into successive surface reactions and it belongs to the class of chemical vapor deposition (CVD) process. Over the last few decades, tremendous progress has made in the variety of materials made by ALD [46] and their applications in related industries [42]. The commonly acknowledged ALD technique was invented back in 1974 under the name "atomic layer epitaxy" (ALE) in Finland by Dr. Tuomo Suntola. The "atomic layer epitaxy" was developed for the growth of high-quality polycrystalline $\mathrm{ZnS}$ thin films used in electroluminescent (EL) flat panel displays. However, the principle of ALD was first introduce in 1960s under the name "molecular layering" in the Soviet Union. In the earlier days ALD did not receive a lot of interest since the film growth process is very slow. The precise controllability of the thickness of the as-grown films combined with the high step coverage for nanostructure fabrication increased the interest toward ALD techniques starting mid 1990's.

ALD is similar to the chemical vapor deposition (CVD) type processes except that it is a repeated process and carried out by breaking a typical CVD process into an iterated sequence of self-saturating deposition cycles [47]. Unlike CVD where the reacting gases are injected and mixed in the process chamber simultaneously to form a film, the ALD reactants or precursors are inserted individually to react with the surface, one layer at a 
time, making it independent of the gas flow distribution. Basic ALD process starts with first introducing a precursor gas onto the substrate surface inside the reactor. A chemisorption process takes place at the surface of the substrate which is referred to an adsorption driven by chemical reaction. This chemisorption process produces a new chemical species covalently bonded to the substrate surface. A finite number of species are deposited on the surface due to the availability of limited number of surface sites which ensures self-terminating process. One of the biggest advantages of the ALD technique is that the exact precursor dosage is not required. The surface saturation limits the reaction; however, enough precursor molecules need to be there to completely cover the substrate surface. The chemisorption process is followed by a purge step where the unreacted precursors and the byproducts are removed with an inert gas $\left(\mathrm{N}_{2}\right.$ or Ar). The second precursor is then introduced into the reactor, which reacts with the first chemisorbed precursor. Excess precursors and the byproducts are removed by another nitrogen purge, which completes the process and creates one monolayer of the final film. The growth per cycle (GPC) is constituted by the sequence of self-limited reactions and purges. Due to the self-terminating reactions in ALD, the thickness of the as-deposited film is controlled by the number of cycles.

\subsection{Synthesis of Tin Selenide ( $\mathrm{SnSe}$ ) using ALD}

Fig. 2.1 shows a flow diagram as an example of how a single layer of SnSe is created using tin bisacetylacetonate $\left(\mathrm{Sn}(\mathrm{acac})_{2}\right)$ and $\mathrm{H}_{2} \mathrm{Se}$ precursors as the reactants. Using this process, we have demonstrated growth of several of the 2D films, including $\mathrm{MoS}_{2}$, 
$\mathrm{WS}_{2}, \mathrm{SnS}$ and $\mathrm{WSe}_{2}, \mathrm{SnSe}$ over a large area $(5 \mathrm{~cm} \times 5 \mathrm{~cm})$. This process can be readily scaled up for industrial applications.

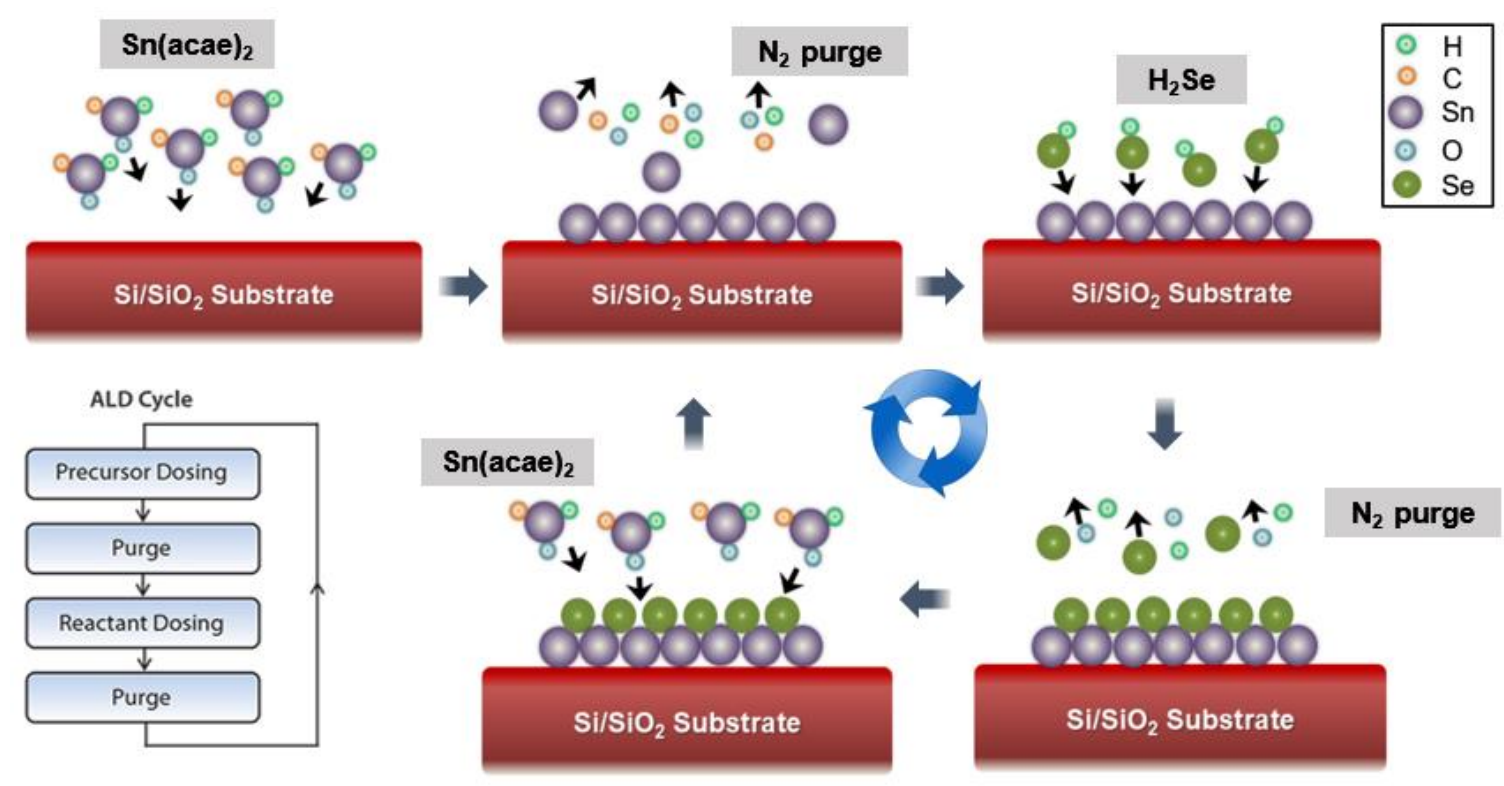

Figure 2. 1: ALD cycle of SnSe.

ALD's self-limiting growth mechanism leads to as-grown thin films with robust and precise atomic level control. The layer-by-layer growth is not affected by gas flow pattern, thus it ensures high degree of uniformity and conformity, making it one of the most promising thin film deposition techniques for nanostructures, patterned substrates, and high aspect ratio features. There are no nucleation based inferior discontinuities in the ALD process, hence the as-grown films uniquely grow pinhole and practically stress free [48]. Chemical Vapor Deposition (CVD) and Physical Vapor Deposition (PVD) techniques are based of nucleation followed by the growth of grains. By the time, the grains finally blend into continuous films the thickness could be on the order of 50-100 $\AA$ [49-50] in the case of CVD, and even thicker in the case of (PVD). CVD and PVD grown films exhibit 
significant compressive stress and lots of pinholes extending far beyond coalescence depth. Pinholes and compressive stress result non-ideal grain boundaries and typically the CVD and PVD films lack passivation. These films yield poor encapsulation performance at any layer where the thicknesses is less than $0.5 \mu \mathrm{m}$. In contrast, very thin encapsulation films can be realized by ALD with minimum adverse impact on device performance. Another characteristic of ALD is that the films can be deposited at low temperatures, which is beneficial for processes where low thermal budget is required. Depending on the film, ALD can deposit films at temperatures as low as $100^{\circ} \mathrm{C}$ which has been demonstrated with $\mathrm{Al}_{2} \mathrm{O}_{3}$ and $\mathrm{ZrO}_{2}$ [51], although most of the TMD films are grown at higher temperatures to produce crystalline films of appropriate phase.

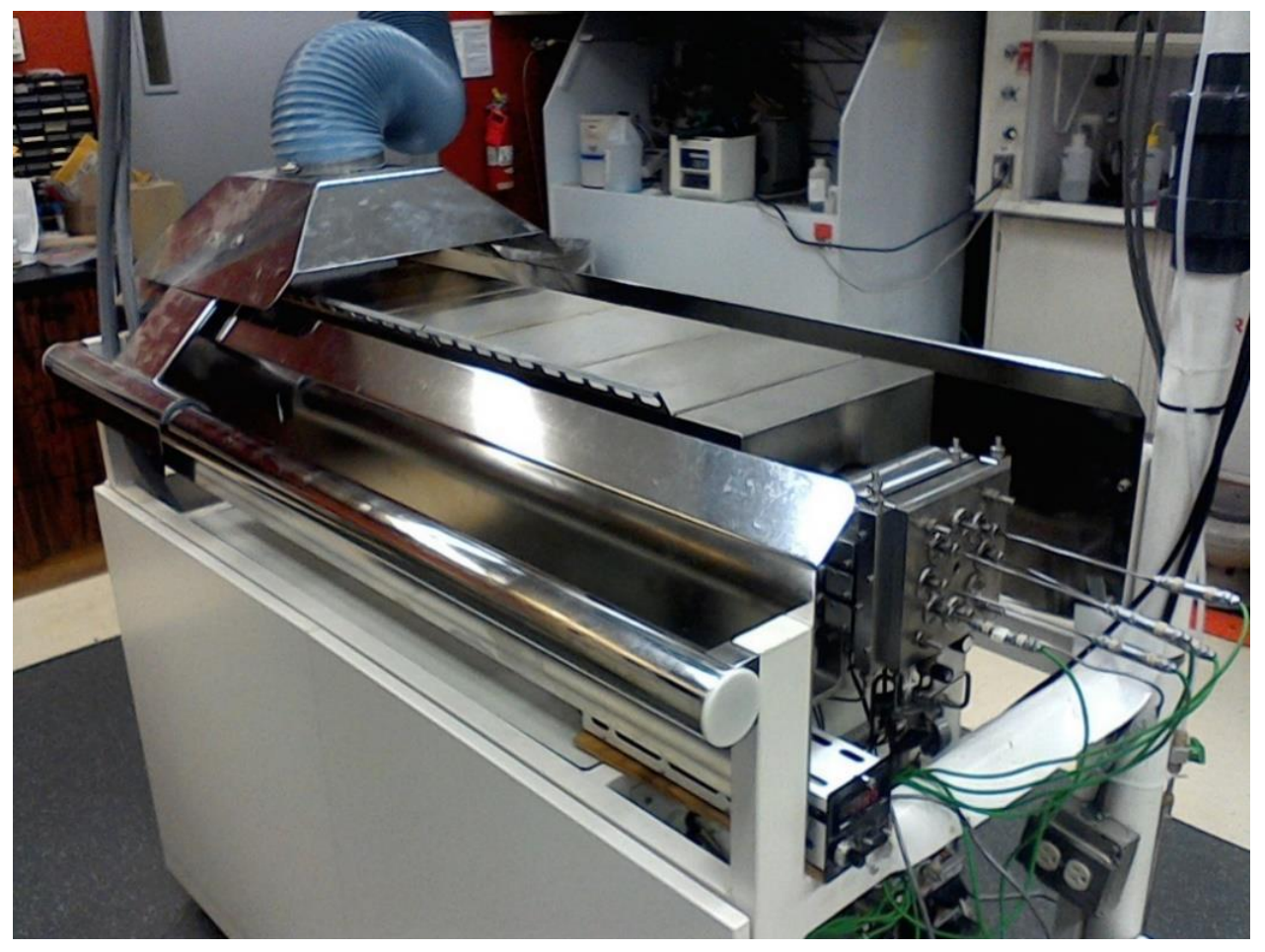

Figure 2.2: Microchemistry F-120 ALD reactor. 
Thin films of SnSe were grown in a Microchemistry F-120 ALD reactor shown in Fig. 2.2 that can handle two $50 \mathrm{~mm}$ x $50 \mathrm{~mm}$ substrates per run. The substrates for these films consisted of p-type Si wafers coated with a $320 \mathrm{~nm}$ thick film of thermal silicon oxide. The Sn precursor was tin (II) acetylacetonate and $\mathrm{H}_{2} \mathrm{Se}\left(8 \%\right.$, balance $\left.\mathrm{N}_{2}\right)$ was the Se source. The exhaust of the system included a burn-box to decompose any unreacted gases. The tin source temperature was held at $95^{\circ} \mathrm{C}$. The growth pulse sequence was as follows: Sn source $0.6 \mathrm{~s}, \mathrm{~N}_{2}$ purge of $1 \mathrm{~s}, \mathrm{H}_{2}$ Se pulse of $1.2 \mathrm{~s}$, and $\mathrm{N}_{2} 1 \mathrm{~s}$ purge. Uniform film growth took place over a temperature ranged from $40^{\circ} \mathrm{C}$ to $410^{\circ} \mathrm{C}$. The films reported here were grown at $390^{\circ} \mathrm{C}$, where the growth rate was $0.5 \mathrm{~nm}$ per cycle. 


\section{CHAPTER 3 \\ Thin Film Characterization}

\subsection{Characterization Techniques}

ALD grown films were characterized using several analytical techniques. Raman spectroscopy measurements were carried out at ambient room temperature on Horiba HR Evolution spectrometer to detect the phase of the films. The optical source for Raman analysis was a doubled YAG laser $(532 \mathrm{~nm})$ and the spectrum was recorded over the range of $40 \mathrm{~cm}^{-1}$ to $300 \mathrm{~cm}^{-1}$. Grazing (0.5 degree) incidence x-ray diffraction (XRD) pattern of the SnSe thin film was recorded with a RIGAKU Ultima IV X-ray diffractometer using $\mathrm{Cu}$ Kalradiation (lambda $=1.5405 \AA$ ) at grazing incidence in the 2 theta range of $10-70$ degrees at room temperature. X-ray photoelectron spectroscopy (XPS) was employed by using Versaprobe II to confirm the stoichiometry of the films. Magnetic property of SnSe was examined with a SQUID system.

\subsection{Raman analysis}

Raman spectroscopy is a popular characterization technique used to identify the stoichiometry or crystalline properties of the materials. It is one of the structural characterization tools that can be used to observe vibrational and other low frequency modes. Inelastic scattering from laser light interaction is the fundamental mechanism of Raman spectroscopy. When laser light interacts with the molecular vibrations or excitations, the energy of the laser photons shifts up or down which provides information regarding the vibrational modes of the system. This is known as Raman Effect. Raman 
spectroscopy measurements were performed to investigate the layered phase and the quality of the as-grown SnSe thin films. The Raman spectrum of the SnSe sample is shown in Fig. 3.1. Five distinct peaks are clearly observed at 69.26, 100.17, 122.59, 151.69 and $184.289 \mathrm{~cm}^{-1}$.

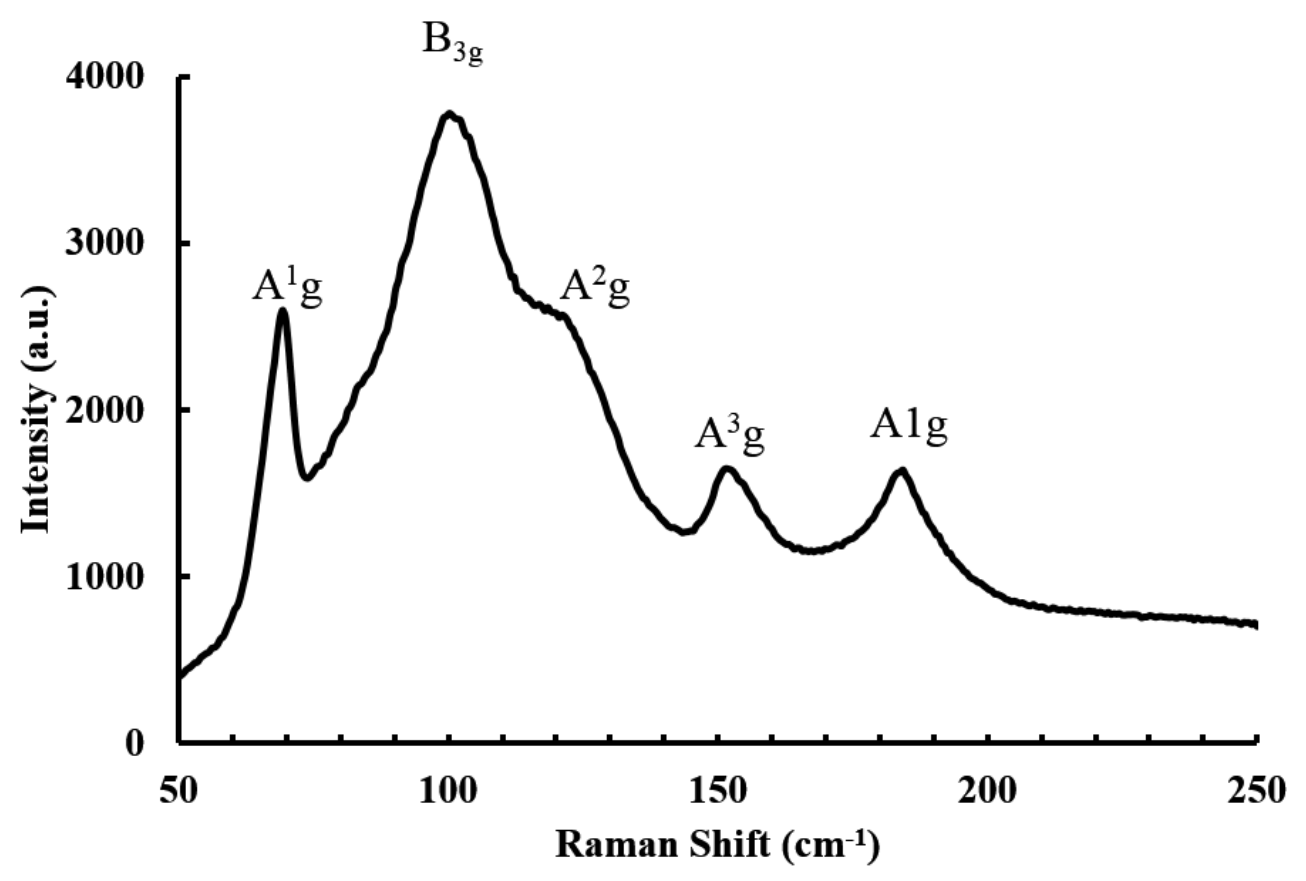

Figure 3. 1: Raman spectrum of as-grown $\mathrm{SnSe}$ thin film on $\mathrm{Si} / \mathrm{SiO}_{2}$ substrate [52].

The highest intensity peak was found at $101 \mathrm{~cm}^{-1}$ which corresponds to the $\mathrm{B}_{3 \mathrm{~g}}$ phonon mode while peaks at 70,123 and $151 \mathrm{~cm}^{-1}$ belong to $\mathrm{A}^{1} \mathrm{~g}, \mathrm{~A}^{2} \mathrm{~g}$ and $\mathrm{A}^{3} \mathrm{~g}$ vibrational modes, respectively. $\mathrm{Ag}$ and $\mathrm{B}_{3 \mathrm{~g}}$ are the two rigid shear modes of a layer with respect to its neighbors and confirm the characteristic planar vibration modes of orthorhombic phase SnSe [53]. The last band A1g at $184 \mathrm{~cm}^{-1}$ could be due to the vibrational mode coming from some trace of $\mathrm{SnSe}_{2}$ as reported by Chitra et al [54]. Although our XRD and XPS 
characterizations did not find any trace of $\mathrm{SnSe}_{2}$, it may be worthwhile to investigate further to determine the source of this peak.

\subsection{X-ray photoelectron spectroscopy (XPS)}

$\mathrm{X}$-ray photoelectron spectroscopy (XPS) is a material analysis technique that measures the elemental composition of the material and provides information regarding interatomic bonds. It can determine the empirical formula, electronic state and chemical state of elements that exist within the material. The XPS spectra typically are acquired by irradiating a material with a beam of $\mathrm{x}$-rays while at the same time determining the kinetic energy and number of electrons escaping from the top to $10 \mathrm{~nm}$ of the material being analyzed. XPS is very useful for metal chalcogenide films analysis to determine the

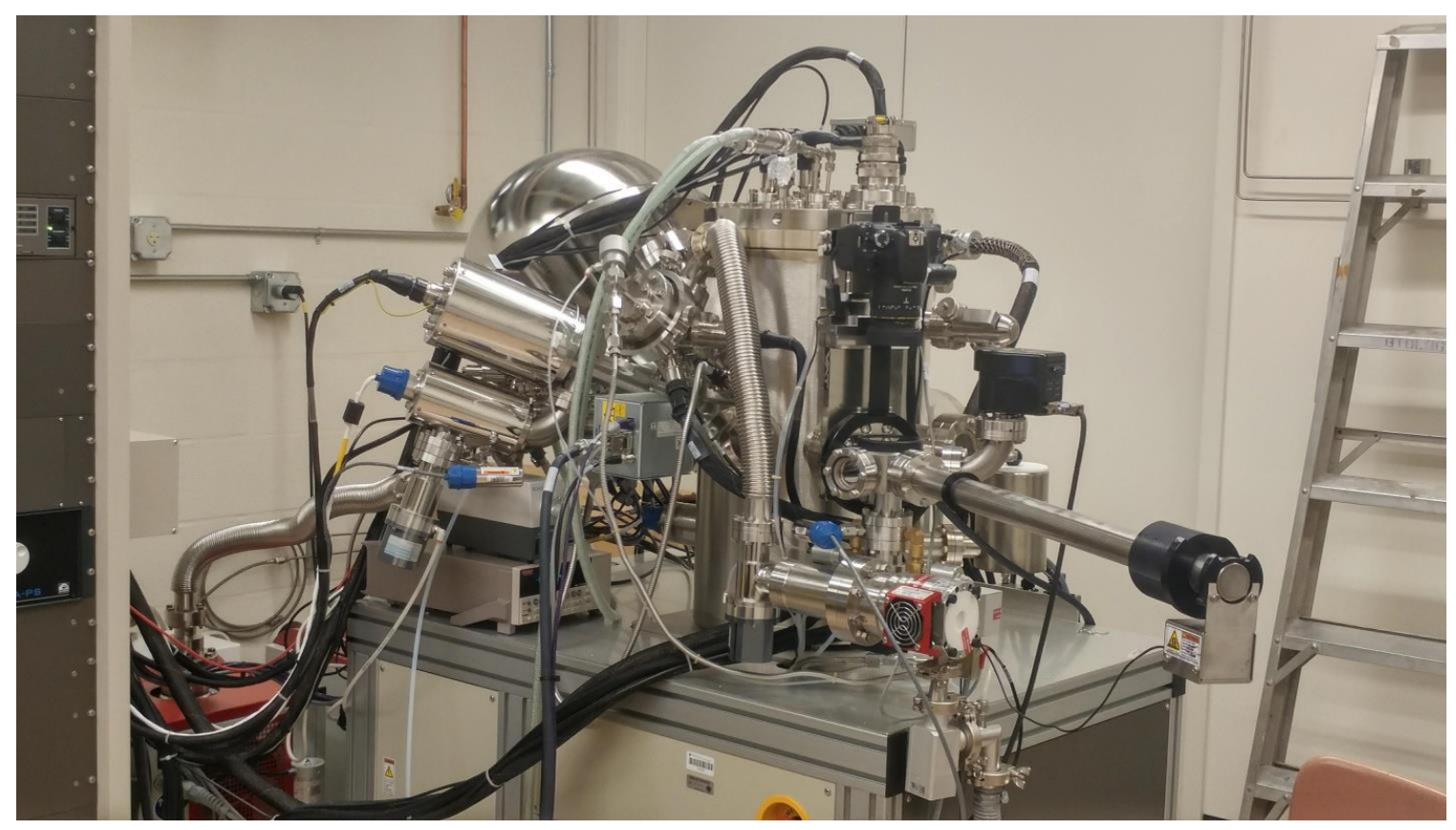

Figure 3. 2: VersaProbe II. 
proper phase and composition of various films as a variant of the growth parameters. It can be used to analyze the surface chemistry of the materials, inorganic compounds, polymers, ceramics, bio-materials etc.
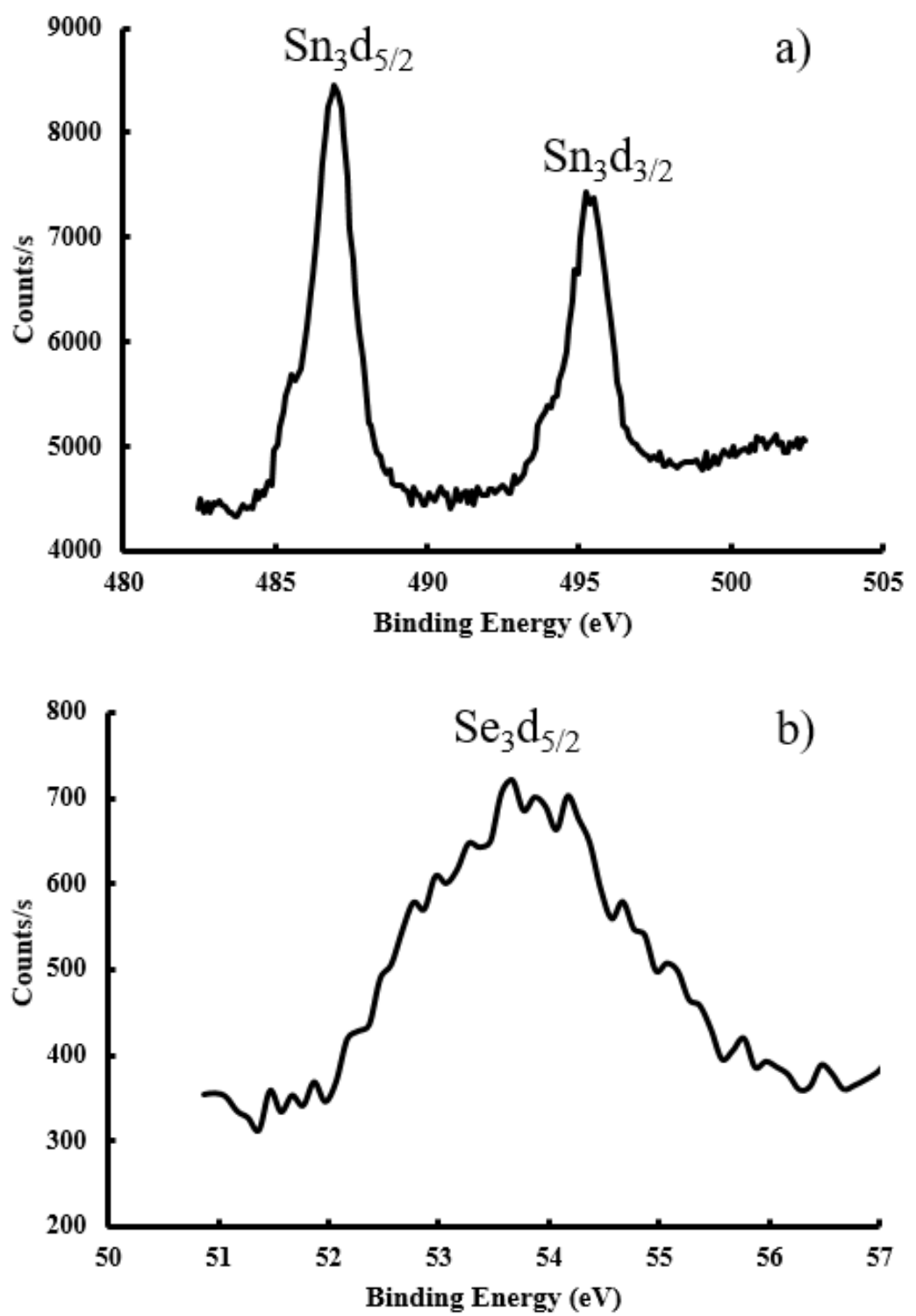

Figure 3. 3: X-ray photoelectron spectroscopy (XPS) on SnSe thin film (a) shows Se 3d and (b) Sn 3d binding energies, respectively [52]. 
XPS of SnSe films was performed using VersaProbe II (Fig 3.2) to determine compositional analysis of the ALD thin films. Fig. 3.3 shows the resulting XPS spectrum of the as-grown SnSe sample. Two strong peaks at approximately 486.2 and $495.7 \mathrm{eV}$ correspond to the $\mathrm{Sn}_{3} \mathrm{~d}_{5 / 2}$ and $\mathrm{Sn}_{3} \mathrm{~d}_{3 / 2}$ state binding energies of $\mathrm{Sn}$, respectively. The Se peak is located at $54.7 \mathrm{eV}$ which corresponds to $\mathrm{Se}_{3} \mathrm{~d}_{5 / 2}$. These results are in close agreement with the earlier reported binding energies for $\mathrm{Sn}$ and $\mathrm{Se}$ in bulk or nanocrystalline $\mathrm{SnSe}$ $[27,55-56]$.

\subsection{X-ray diffraction (XRD)}

X-ray diffraction (XRD) is a non-destructive characterization tool that can be used to identify various materials and their crystalline properties such as minerals, inorganic compounds, fine-grained minerals, clays and mixed layer clays, which are difficult to determine optically. Other applications include obtaining phase, orientation and unit cell dimensions of the materials, structural properties such as lattice parameters (interplanar spacing), strain, grain size, epitaxy, and phase composition. It can also be used to determine the thickness of thin films, samples purity, multilayers and atomic arrangement. 


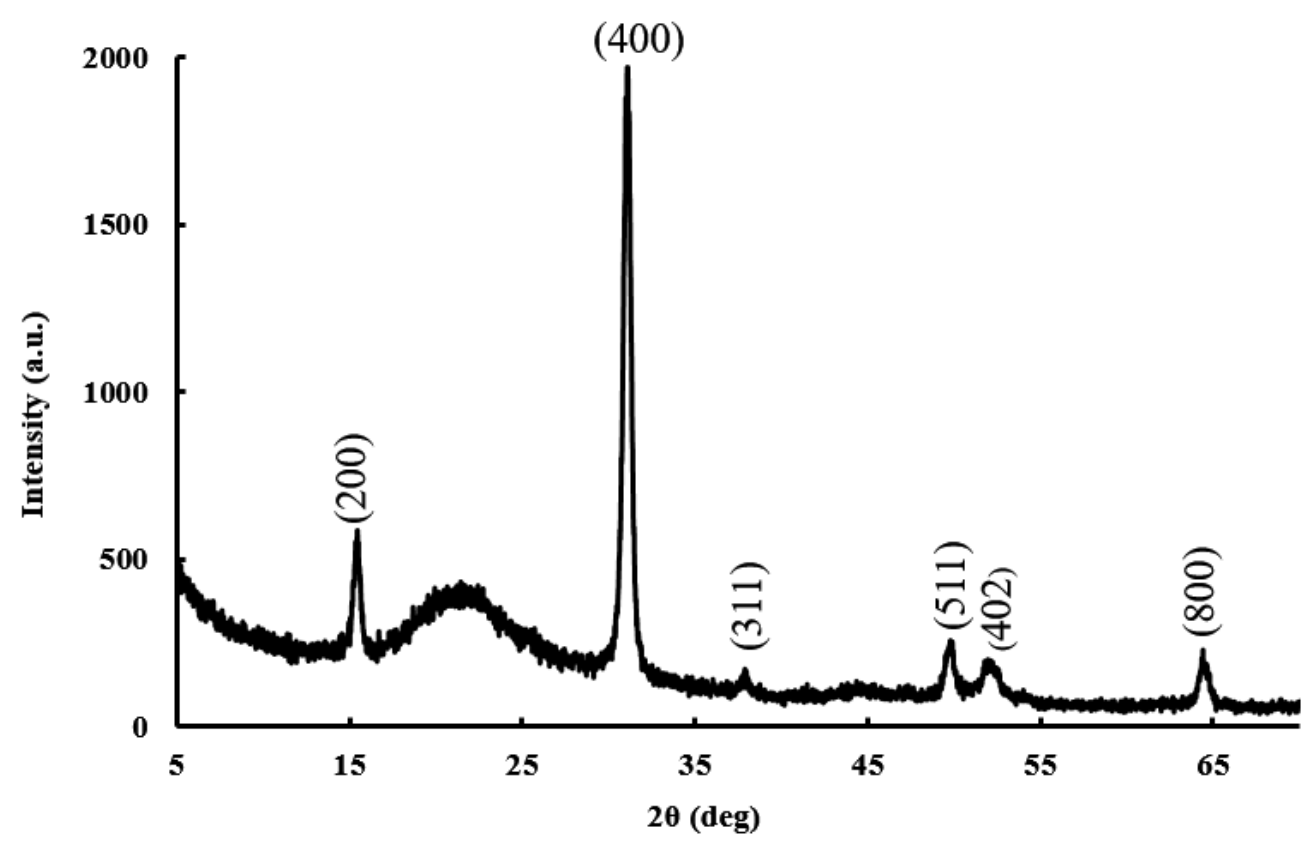

Figure 3. 4: X-Ray diffraction pattern of SnSe thin film grown by ALD [52].

In this research, X-ray diffraction (XRD) was performed to investigate the phase structure of SnSe thin films. Grazing incident X-ray beam angle was kept very small (0.5 degree) to maintain the background scattering as low as possible from the amorphous $\mathrm{SiO}_{2}$ substrate. This technique is particularly helpful for thin film XRD characterization where the penetration depth of the X-ray is important. Grazing incidence XRD pattern from the as-grown SnSe shown in Fig. 3.4 was recorded at room temperature. Formation of well crystallized films is also evident from the sharp peaks. The XRD pattern shows the presence of sharp peaks corresponding to (200), (400), (311), (511), (402) and (800) planes of SnSe. It is worthwhile to note that the predominant peaks are (400) and (200). These peaks are consistent with the peaks of orthorhombic SnSe reported in literature synthesized by various techniques [7, 27-28, 
$37,57]$. A broad XRD peak located approximately at $22.5^{\circ}$ is from the amorphous $\mathrm{SiO}_{2}$ film on the silicon substrate [58].

\subsection{Magnetic property}

Magnetic properties of these films were characterized on a superconducting quantum interference device (SQuID). The system used for this technique allowed samples to be inspected over a temperature range from room temperature to $1.8 \mathrm{~K}$, and magnetic fields of -7 to 7 teslas. The magnetic moment (emu) vs applied field $(\mathrm{Oe})$ plot measured at $1.8 \mathrm{~K}$ is shown in (Fig. 3.5). After subtracting the Si substrate diamagnetic background, the thin SnSe film shows a paramagnetic profile which suggests that there are unpaired electrons attracted by the magnetic field due to the electrons' magnetic dipole moments.

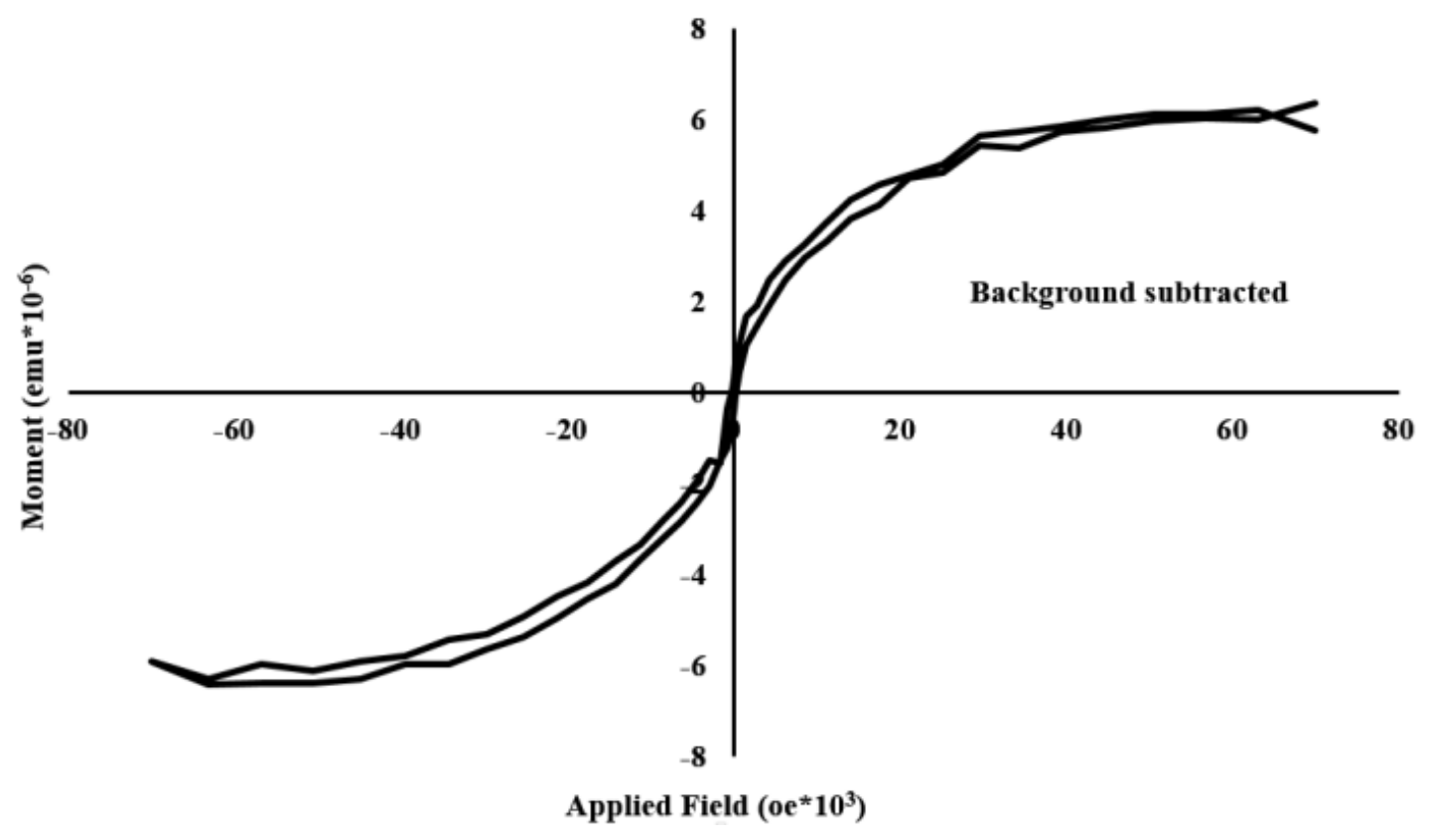

Figure 3. 5: Magnetic properties of thin film SnSe illustrating a paramagnetic profile [52]. 


\subsection{Optical Properties}

Tin selenide, due to its narrow band gap and high optical absorption coefficient, is a material of interest for optoelectronics and photovoltaic applications [12]. However the electronic structure and physical properties are affected by the dimension and phase of these materials. As reported by Guangsha et al. different layer thickness of p-type SnSe thin films exhibit different values of bandgap energy [59]. In the case of bulk orthorhombic tin selenide ( $\mathrm{SnSe}$ ) the direct bandgap is $1.3 \mathrm{eV}$ whereas $1.66 \mathrm{eV}$ and $1.62 \mathrm{eV}$ respectively for single-layer and double-layer as illustrated in Fig. 3.6. Quantum confinement effect is responsible for the variation in bandgap, thus direct bandgap of SnSe thin films is greater than that of bulk SnSe [60]. N. Kumar et al. reported that bandgap increases monotonically with the decrease of film thickness [61]. Furthermore, experiments demonstrate that the derived optical bandgap of bulk orthorhombic SnSe is less than that of bulk cubic SnSe (SnSe-CUB) [62-63]. As reported in the literature for solution-phase synthesis, SnSe nanocrystals show a direct bandgap of about $1.71 \mathrm{eV}$ whereas SnSe nanowires exhibit $\sim 1.55 \mathrm{eV}$ direct bandgap [60,64]. The variation of the bandgap energy (in $\mathrm{eV}$ ) for different structures of orthorhombic SnSe is summarized in Table 1.

\begin{tabular}{|c|c|c|c|c|c|}
\hline Bandgap & Nanocrystal & $\begin{array}{c}\text { Single } \\
\text { layer }\end{array}$ & $\begin{array}{c}\text { Double } \\
\text { layer }\end{array}$ & Nanowires & Bulk \\
\hline Direct & 1.71 & 1.66 & 1.62 & 1.55 & 1.3 \\
\hline
\end{tabular}

Table 1: Optical bandgap energies of different thickness of orthorhombic SnSe in eV [65].

The band structures of single-layer, double-layer and bulk SnSe are shown in Fig. $3.6(\mathrm{a}-\mathrm{c})$. The distance between the valence band maximum (VBM) point and conduction 
band minimum (CBM) point indicates the energy band gap. For single-layer and doublelayer structures, the conduction band minima are located along both the $\Gamma-\mathrm{X}$ and $\Gamma-\mathrm{Y}$ directions while the valence band maximum is along $\Gamma-Y$. Thus, minimum direct gap in single-layer and double-layer SnSe is located along $\Gamma$-Y. In bulk SnSe, the conduction band minimum is located along $\Gamma-\mathrm{X}$, whereas the valence band maximum is along the $\Gamma-\mathrm{Y}$ direction. The smallest direct gap is $1.30 \mathrm{eV}$ for bulk and located along the $\Gamma-\mathrm{X}$ direction [59].

(a)

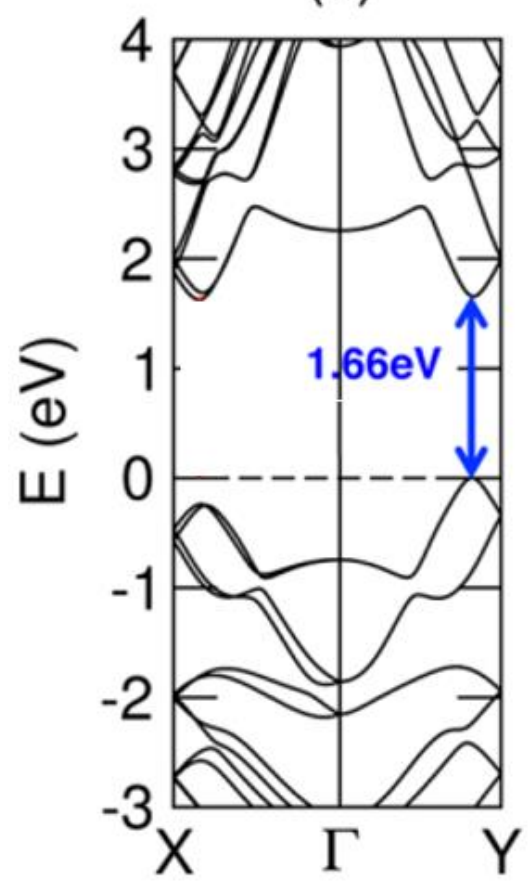

(b)

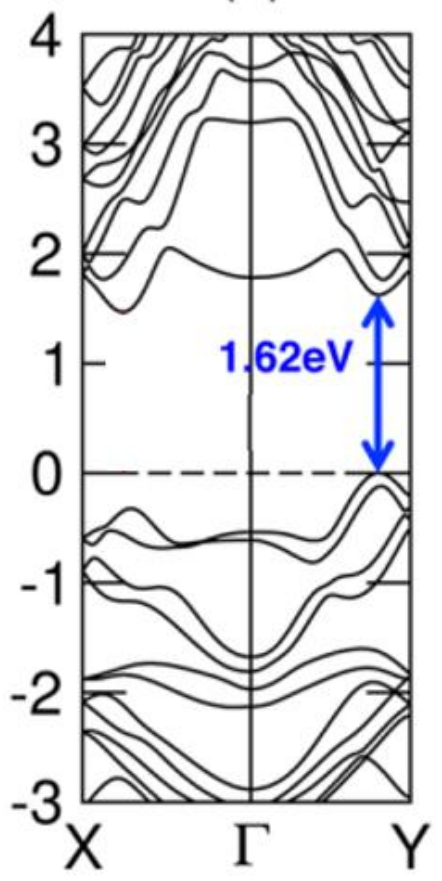

(c)

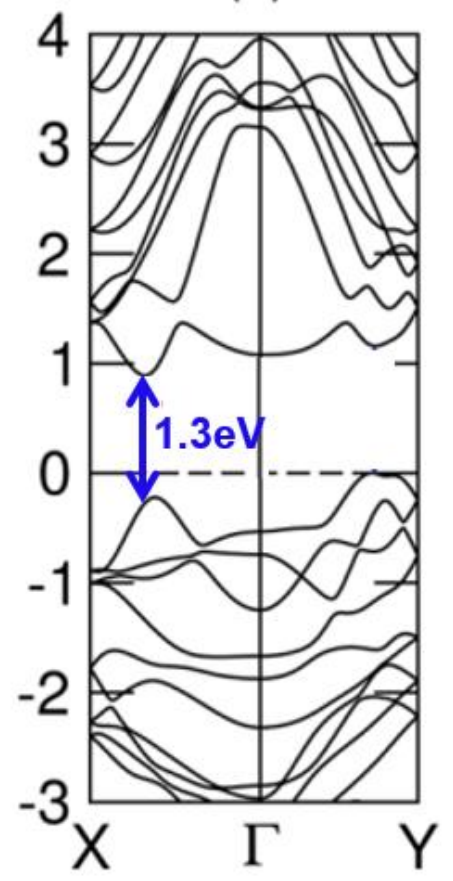

Figure 3. 6: Optical band structures of a) single-layer, b) double-layer, and c) bulk SnSe. Arrows indicate direct transitions [59].

Bandgap energy is one of the primary factors for any material to be considered for optoelectronics and photovoltaic applications. The bandgap energy of the ALD-grown 2D layered SnSe samples were characterized by UV-Vis spectroscopy. The bandgap energy 
(Eg) was determined from the absorption spectra of the films grown on glass plates. The direct bandgap was calculated from extrapolation of the linear plot of $(\alpha h v)^{2}$ versus photon energy ( $h v$ ) onto the x-axis directly (shown in Fig. 3.7) following the Tauc formula:

$$
(\alpha h v)^{2}=k(h v-E g)
$$

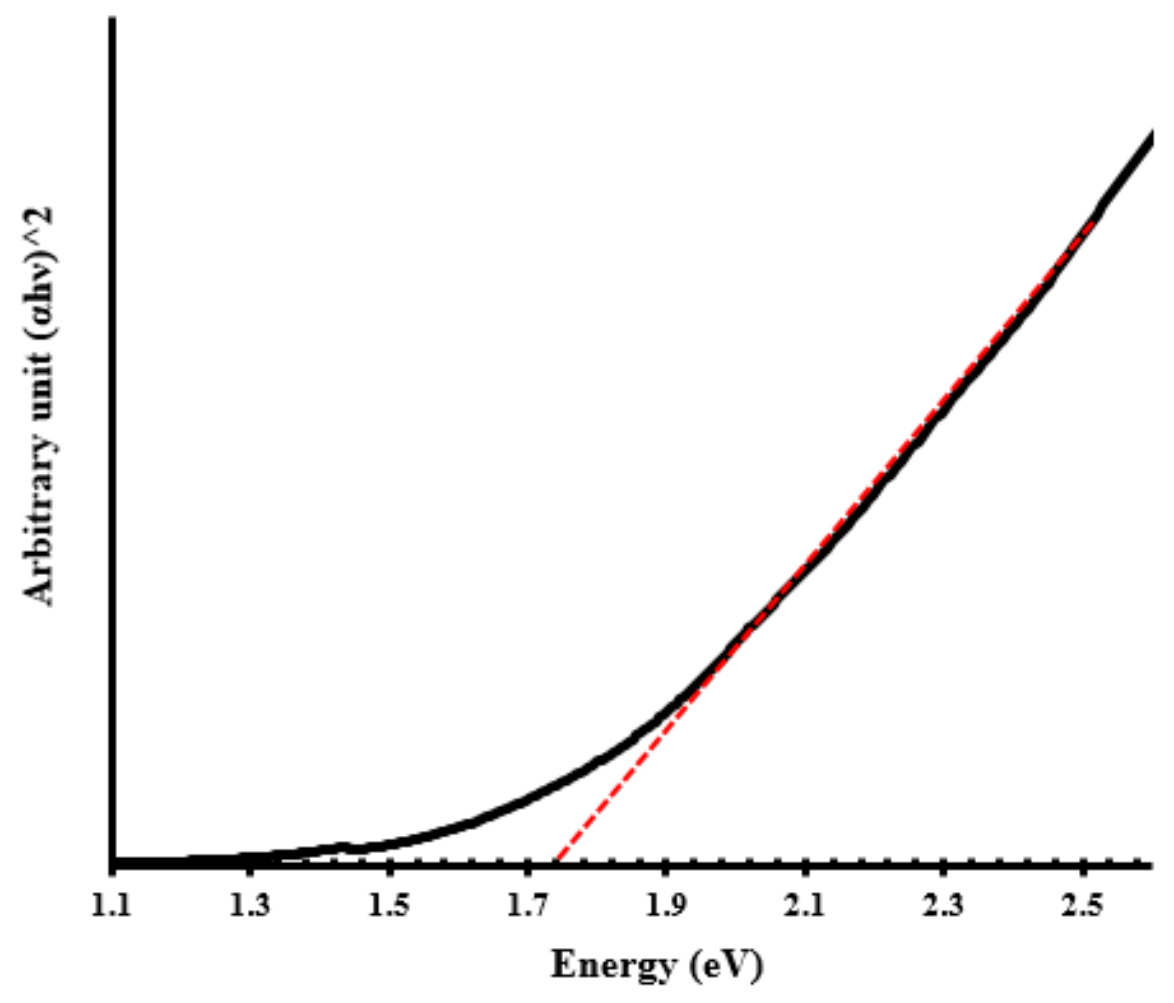

Figure 3. 7: Energy band gap calculation of ALD synthesized SnSe thin film.

Where $\alpha, h$ and $v$ are the absorption coefficients, Planck constant and light frequency, respectively. The linear behavior of the plot observed at high energy region indicates band to band direct transitions in these films. The band gap energy was estimated to be about $1.74 \mathrm{eV}$ for the SnSe synthesized thin film samples. This value is in close agreement with the reported values for SnSe nanocrystals deposited by other techniques [60]. 


\section{CHAPTER 4}

\section{Device fabrication and electrical characterization}

\subsection{Background}

Digital and analog electronic circuits are generally composed of metal-oxidesemiconductor field-effect transistor (MOSFET) as the fundamental block. In logic circuits, these transistors constitute large assemblies of logic gates where each of the transistor in the logic gates has two distinct on and off states of conductance differentiated by several order magnitude difference in the channel current. Unlike in gapless graphene, 2D metal chalcogenide materials have band gaps greater than $1 \mathrm{eV}$ which makes them ideal candidates for electronic circuits requiring large on/off current ratios. Tin selenide (SnSe) exhibits a direct bandgap of $1.3 \mathrm{eV}$, a good candidate for applications in photovoltaics and optoelectronics devices. Due to its polarity dependent electronic properties, single crystal SnSe has potential applications in memory devices. A few monolayer thick SnSe films possess excellent photosensitivity and tunable bandgap over bulk form due to the higher surface to volume ratio and quantum confinement effects. To investigate the electronic properties of the ALD grown SnSe thin films, several back-gated FET transistors were fabricated using optical lithography. 


\subsection{Photolithography}

The 2D semiconductor SnSe films were grown on p-type doped (100) silicon substrates with $320 \mathrm{~nm}$ thick thermally grown $\mathrm{SiO}_{2}$. The SnSe film thickness was typically 5-6 nm depending on the number of ALD growth cycles. The different layers of the wafer are shown in Fig. 4.1.

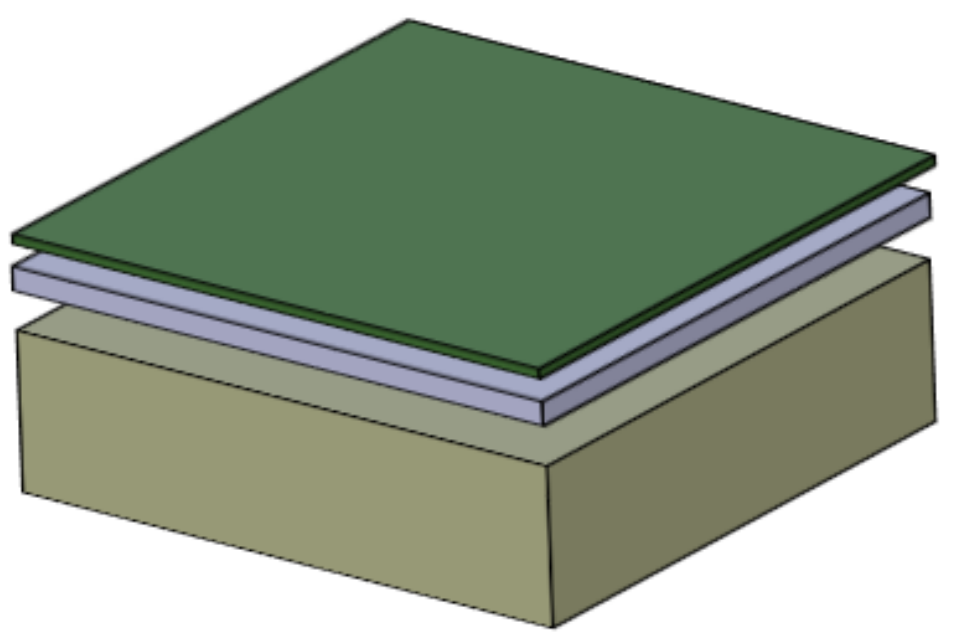

\section{$\operatorname{SnSe}(5-6 \mathrm{~nm})$} $\mathrm{SiO}_{2}(320 \mathrm{~nm})$

Si Substrate $(625 \mu \mathrm{m})$

Figure 4. 1: Schematic of ALD synthesized $\mathrm{SnSe}$ thin film on $\mathrm{Si} / \mathrm{SiO}_{2}$ substrate.

A photo-mask was used for pattering the wafers to define source and drain contacts.

The contact mask used for the fabrication was a 5inch $\mathrm{x}$ 5inch soda lime glass mask is shown in Fig. 4.2. The mask was designed using AutoCad and then commercially fabricated. The mask was designed to pattern transistors of gate lengths $2 \mu \mathrm{m}, 4 \mu \mathrm{m}, 6 \mu \mathrm{m}$, $8 \mu \mathrm{m}$, and $10 \mu \mathrm{m}$. All the transistors had gate width of $100 \mu \mathrm{m}$. The mask also included TLM structures to measure the contact and sheet resistances of the SnSe film. 


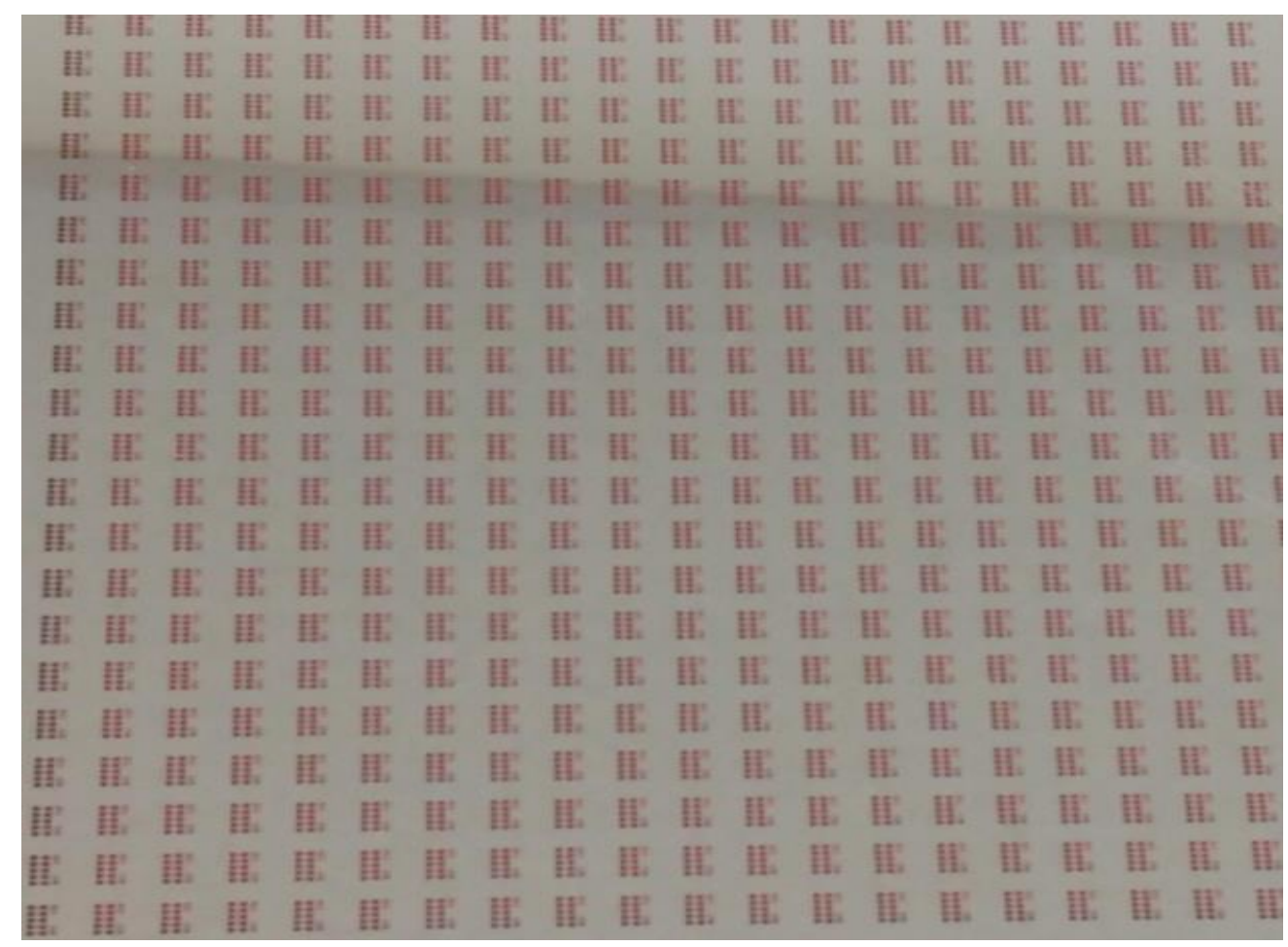

Figure 4. 2: Contact Mask for patterning.

The wafer was cleaned using standard photolithography cleaning process using solvent (Acetone, Methanol and IPA) followed by DI (deionized water) rinse and $\mathrm{N}_{2}$ blow dry. Shipley Microposit S1813 positive photoresist was used to coat the wafers in order to pattern the features. To achieve the resist film thickness around 1.5 micrometer, the spin coater spinning (shown in Fig. 4.3) was set at 3200 RPM for 110 seconds. 


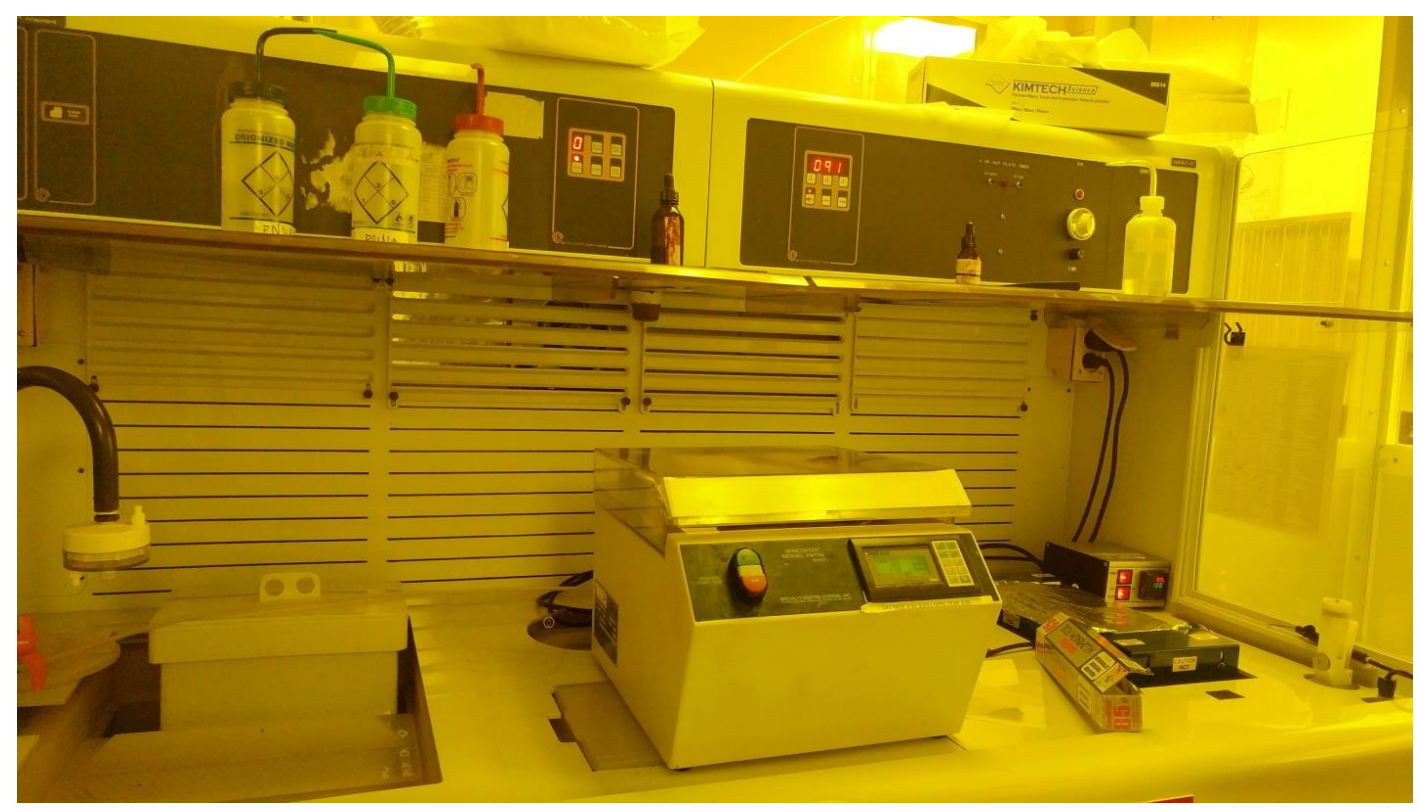

Figure 4. 3: Spin coater P6700 used to coat the wafer using photoresist.

The resist was soft baked at $100^{\circ} \mathrm{C}$ on a hotplate for around 60 seconds to improve adhesion and prevent the stickiness with the contact mask. The resist coated wafers were patterned and exposed using the OAI Hybralign 200 Contact Aligner (shown in Fig. 4.4) with UV light of $365 \mathrm{~nm}$ wavelength. The dose was optimized for 120 seconds. 


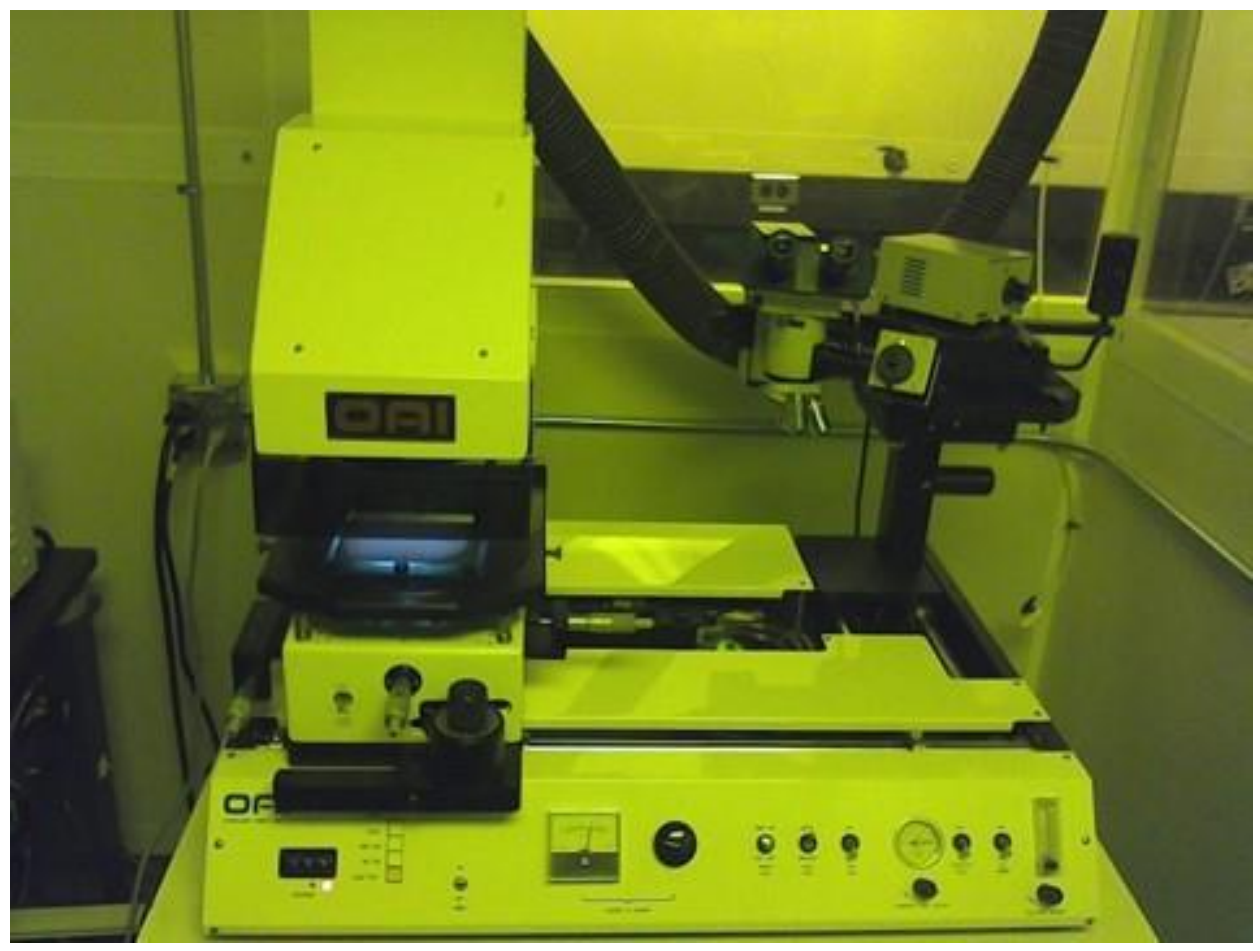

Figure 4. 4: OAI Hybralign 200 contact Aligner used for Lithography exposure.

$\mathrm{NaOH}$ was used as the developer solution with a concentration between 0.133 and 0.194 M for removing the exposed photoresist from the wafers. This was followed by DI water rinse and blow dry. It is noteworthy to mention that the concentration of $\mathrm{NaOH}$ solution was varied and optimized in order to achieve good pattern uniformity across the wafers. Fast development causes poor uniformity which may create overdeveloped structures in the center and underdeveloped at the edges of the wafers. The final optimized development time was set approximately between 15 to 20 seconds. The developed patterns were verified using optical microscope shown in Fig. 4.5. The next process was metal deposition on the patterned substrates which was performed via sputtering. 


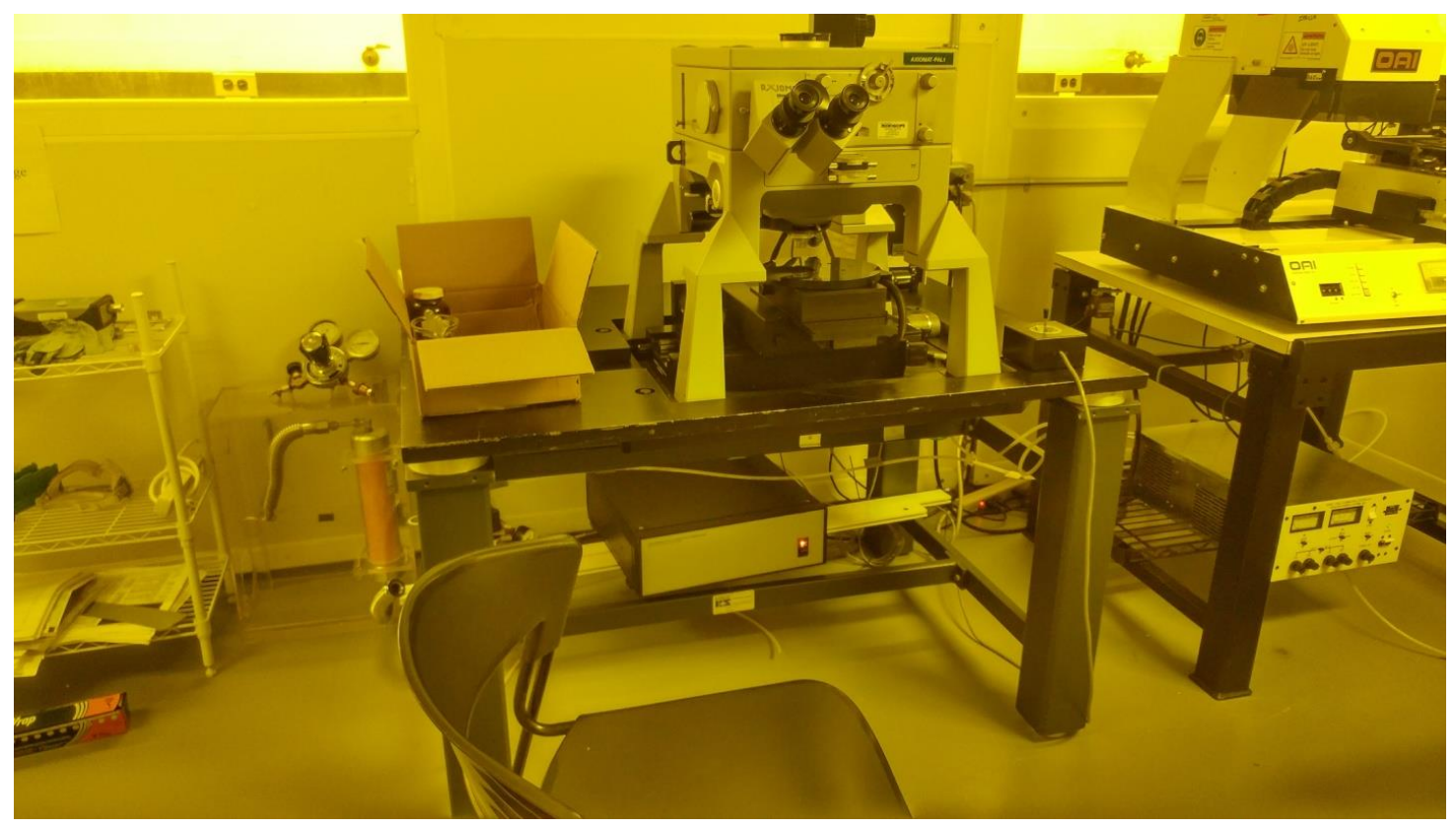

Figure 4. 5: Optical Microscope used for imaging at different steps of the fabrication.

Metal deposition was employed to produce source/drain contacts. Au was selected as the source/drain metal because of its large work function $(\sim 5.3 \mathrm{eV})$. This film was produced via sputter deposition. The thickness of the Au films on the different substrates was around 30 to $60 \mathrm{~nm}$. After the metal deposition, the unexposed photoresist was lifted off using acetone followed by DI rinse and blow dry. An Ag paste was applied to the backside of the wafers to provide backside metal contact. The schematic of the back-gated transistor with source, drain and gate contacts are shown in Fig. 4.6 (a). Optical image for one of the final fabricated SnSe samples with array of active transistors are shown in Fig. 4.6 (b). Fig. 4.6 (c) shows one set of transistors with varying gates lengths and associated TLM structures (details in next section). 


\section{Source/Drain: Au}

(a) Gate: Ag
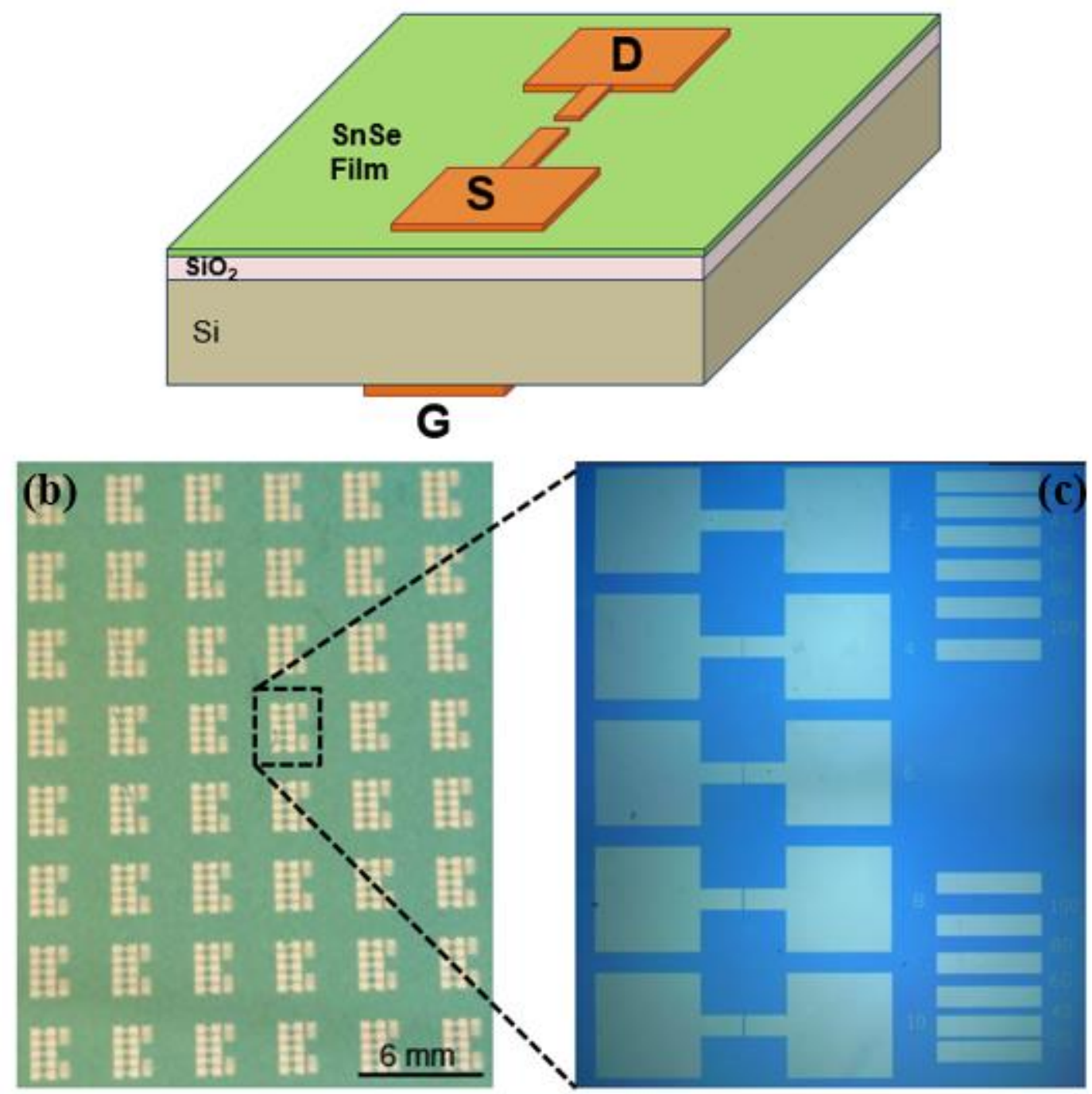

Figure 4. 6: a) Schematic diagram of a SnSe back-gated transistor, b) patterned features on the substrate after metallization showing array of devices, c) optical view of the patterned substrate focusing on a cluster of transistors of gate lengths of $2,4,6,8$, and $10 \mu \mathrm{m}$ and gate width of $100 \mu \mathrm{m}$. 


\subsection{Test of electrical properties}

Electrical characterization is one of the most important factors to understand the electronic properties of the semiconductor materials. To investigate the electrical properties, drain current-voltage $\left(I_{d}-V_{d}\right)$ and drain current-gate voltage $\left(I_{d}-V_{g}\right)$ scans were performed by using back gated filed effect transistors in order to extract basic electrical information such as carrier mobility, on/off current ratio and contact parameters.

Electrical tests were performed by using a probe station (MODEL 7000-LTE) shown in Fig. 4.7, which was connected to HP 4145B semiconductor parameter analyzer (Fig. 4.8). The parameter analyzer was interfaced to a workstation through serial port and could be controlled by characterization software ICS (Interactive Characterization Software). This software provided the inputs to the analyzer as well as recorded the output parameters for every test and stored the data and the plots.

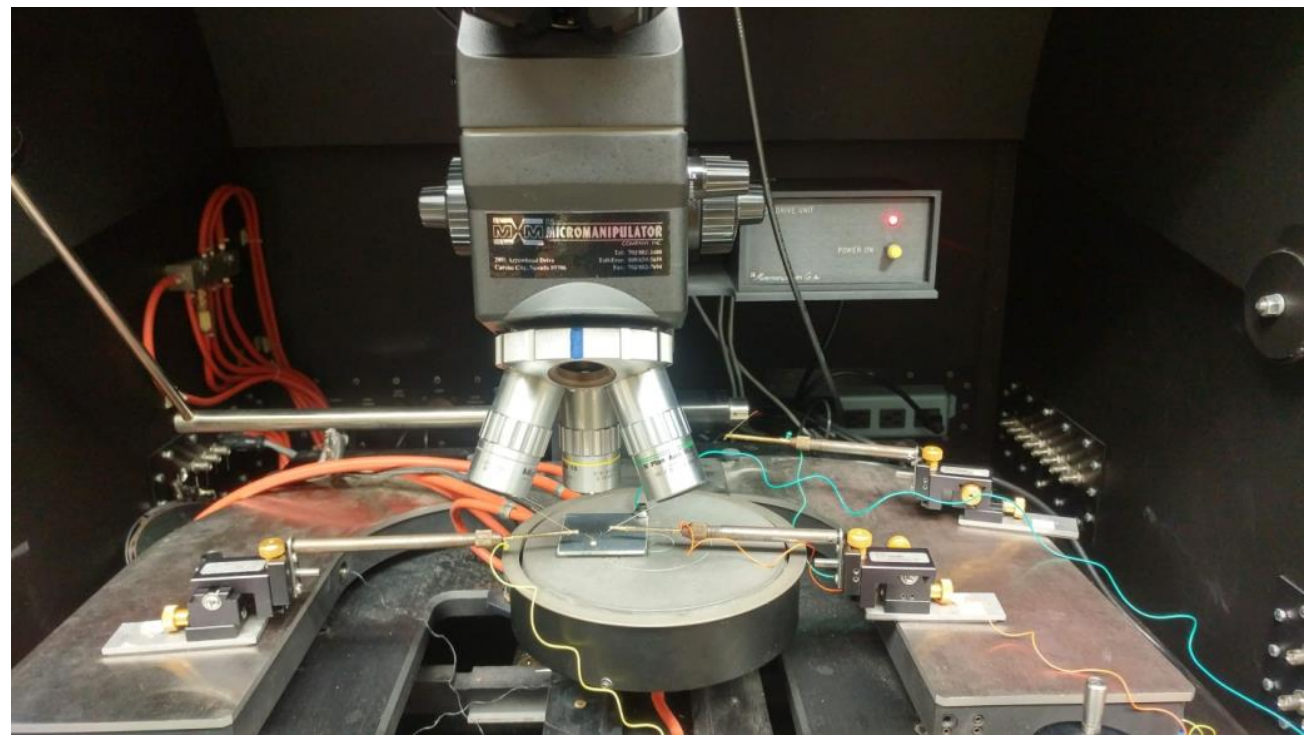

Figure 4. 7: Probe Station used for electrical testing. 


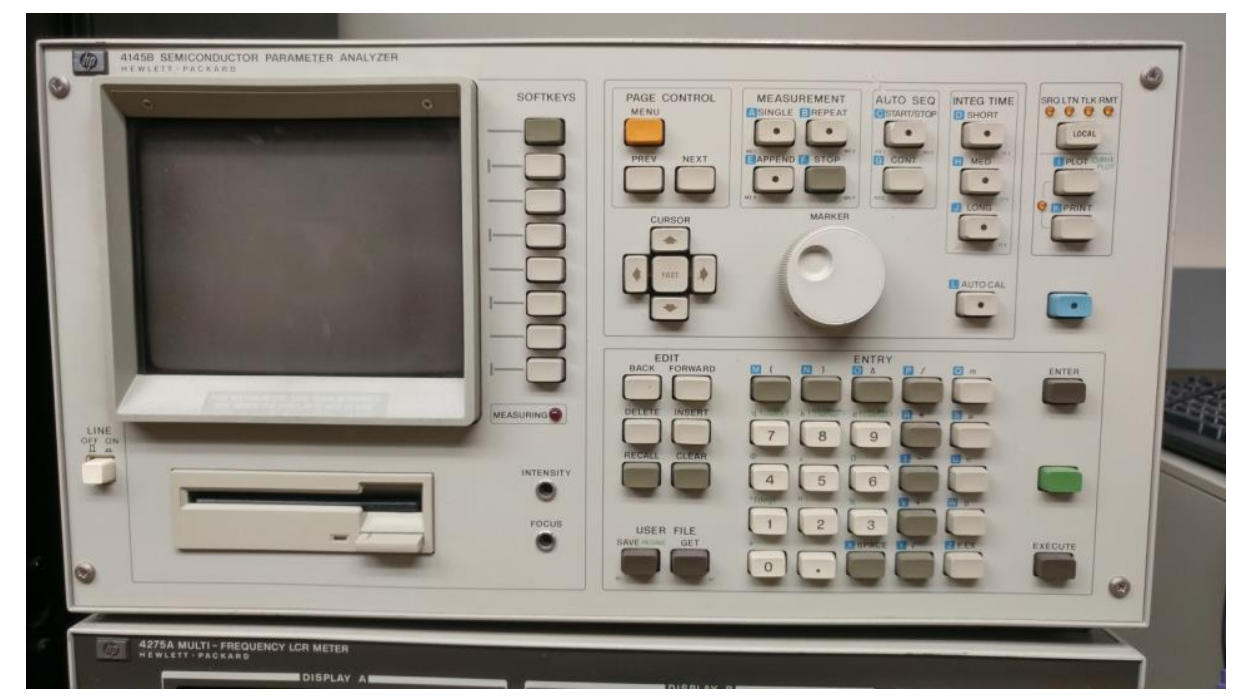

Figure 4. 8: HP 4145B Semiconductor Parameter Analyzer.

Electrical proprieties of the as-synthesized SnSe were investigated by fabricating back-gated field-effect transistors (FETs) using standard optical lithography techniques as described in section 4.2. Each cluster consisted of multiple transistors of varying gate lengths with a fixed gate width of $100 \mu \mathrm{m}$ as shown in Fig. 4.6 (b).

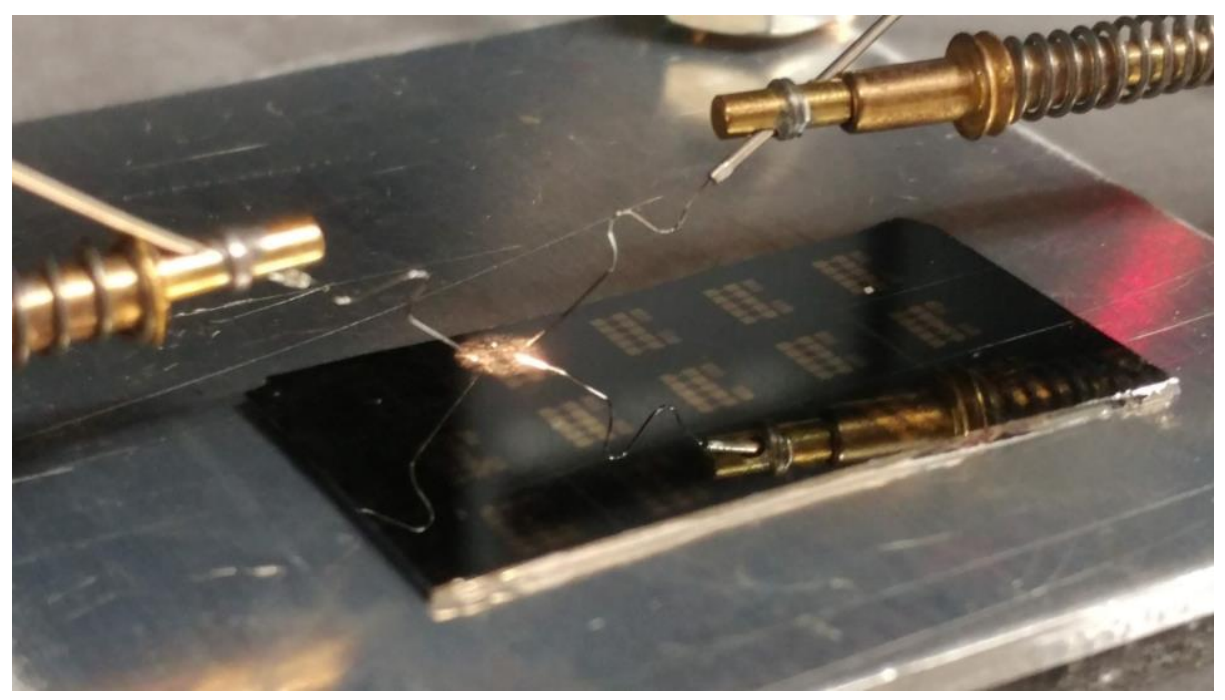

Figure 4. 9: Electrical probing of the back gated SnSe transistors. 
The fabricated FETs were characterized at room temperature (Fig. 4.9). With zero gate bias, the drain current versus drain voltage exhibited linear profile indicating ideal ohmic contacts between Au electrodes and SnSe (Fig. 4.10).

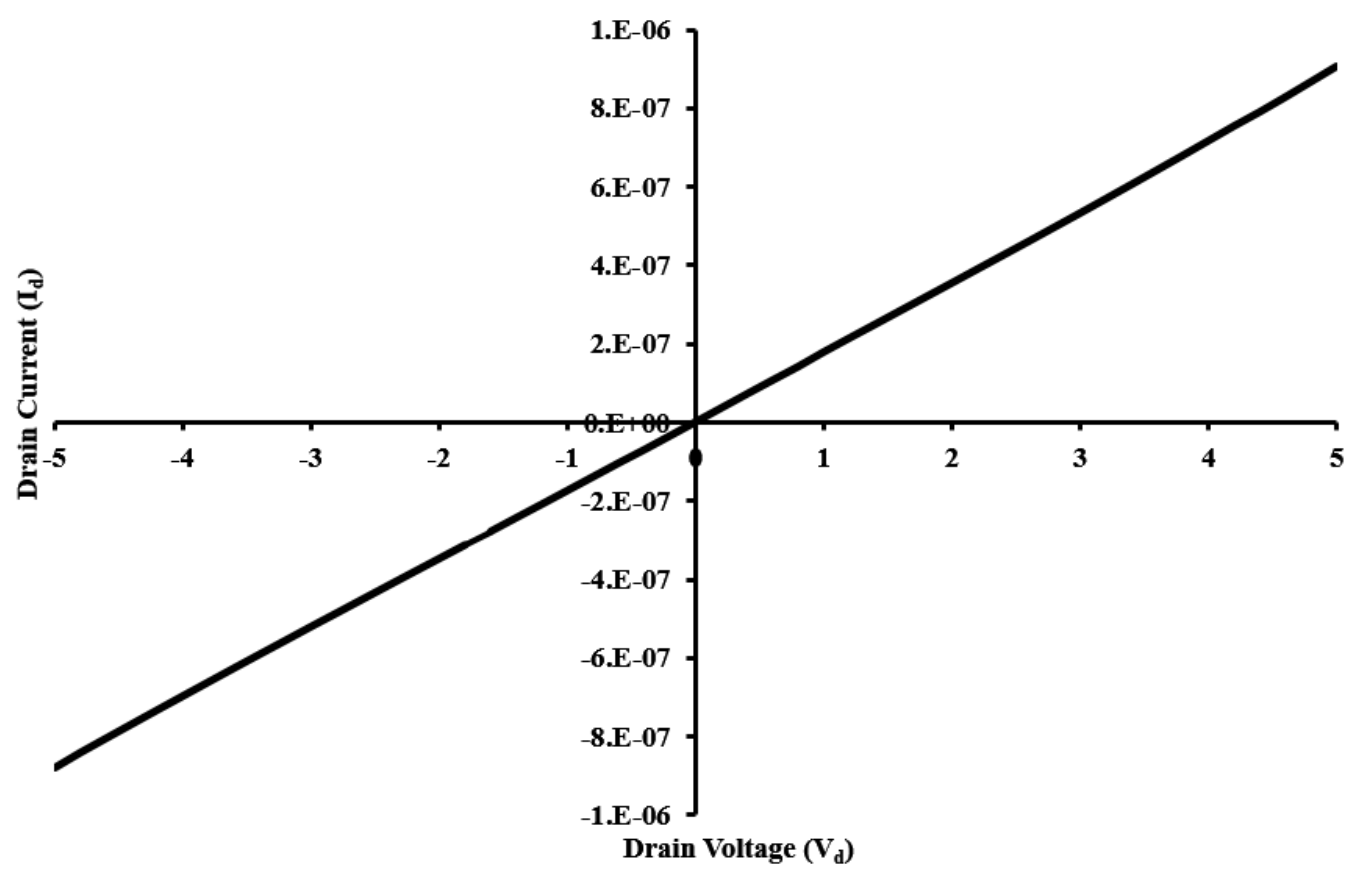

Figure 4. 10: Linear $I_{d}-V_{d}$ characteristic of SnSe thin film with Au electrodes at zero gate bias [52].

Prior to each transistor characterization, I-V curves were plotted between S/D and back gate to detect onset of leakage. For subsequent transistor characterization, gate voltages were maintained much lower than this value in order to prevent measurement of leakage current. Also, a trench was scribed around each transistor to eliminate leakage across the edge of the substrate. The photo-response of the as-grown SnSe thin films was also characterized using the FET device by turning the illumination on and off as shown in [Fig 4.11]. Drain current increases with illumination which implies that the SnSe thin film exhibits direct band gap. 


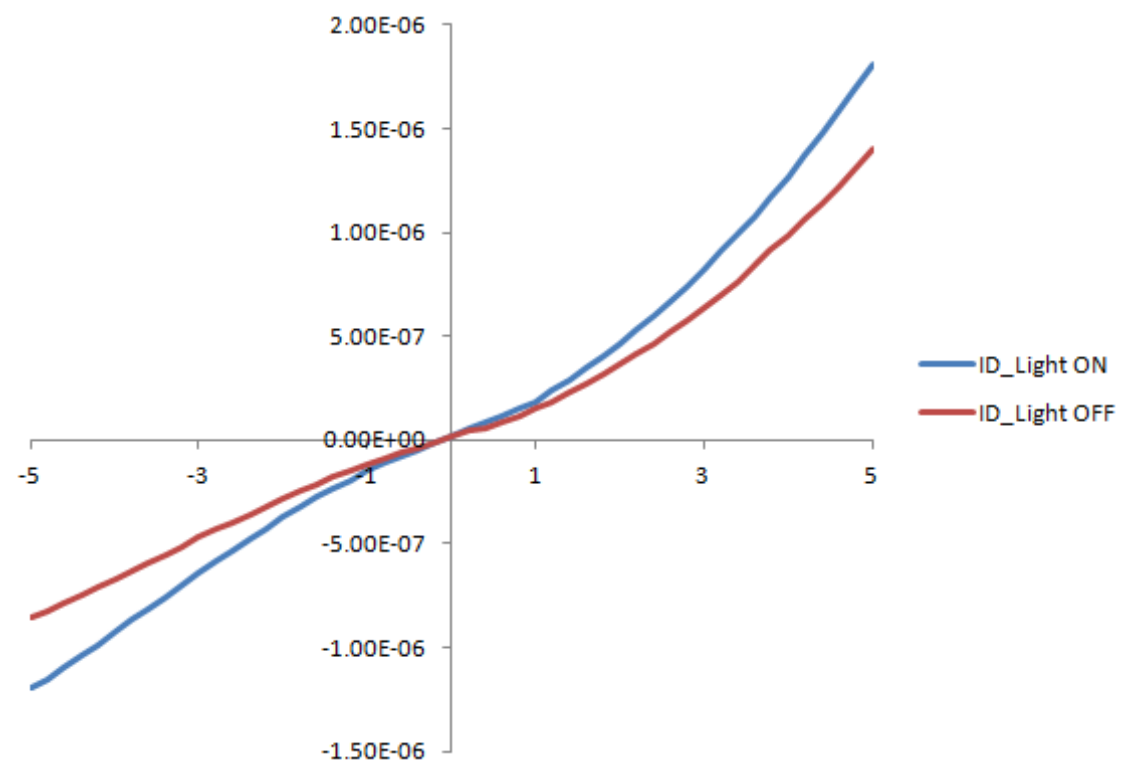

Figure 4. 11: Current-voltage characteristics of a SnSe FET under illumination at zero gate bias.

Transfer characteristics were determined by keeping the drain voltage $\left(\mathrm{V}_{\mathrm{ds}}\right)$ constant at $0.1 \mathrm{~V}$ while varying the back-gate voltage $\mathrm{V}_{\mathrm{g}}$ to the $\mathrm{Si}$ substrate from 0 to $-15 \mathrm{~V}$.

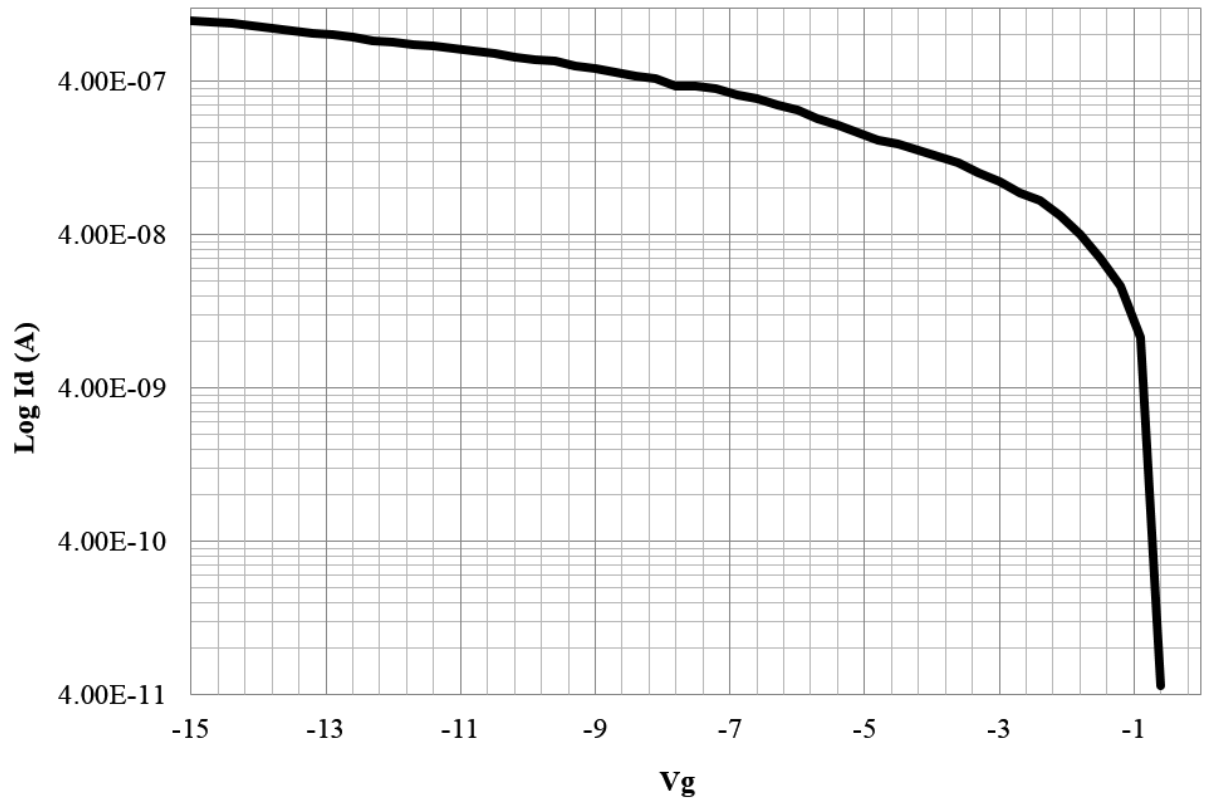

Figure 4. 12: Transfer characteristics of a SnSe p-FET [52]. 
The $\mathrm{I}_{\mathrm{d}}-\mathrm{V}_{\mathrm{g}}$ transfer characteristic of the device showed p-type transistor behavior as can be seen in (Fig. 4.12). Drain current varied with the gate voltage corresponding to an on/off current ratio of nearly $10^{5}$ which is well within the desired $\mathrm{I}_{\text {on }} / \mathrm{I}_{\text {off }}$ ratio of $10^{4}-10^{7}$ for any silicon replacement FET [66]. The field effect mobility was calculated using the following equation:

$$
\mu=\left(\Delta I_{d} / \Delta V_{g}\right) *\left(L / W C_{S i O 2} V_{d}\right)
$$

Where $\mathrm{L}$ and $\mathrm{W}$ are the length and width of the device, and $\mathrm{CSiO}_{2}=11 \mathrm{nF} / \mathrm{cm}^{2}$ is the capacitance for $320 \mathrm{~nm} \mathrm{SiO} 2$ layer. The average room temperature mobility of several of these devices were about $10 \mathrm{~cm}^{2} /(\mathrm{V} . \mathrm{s})$.

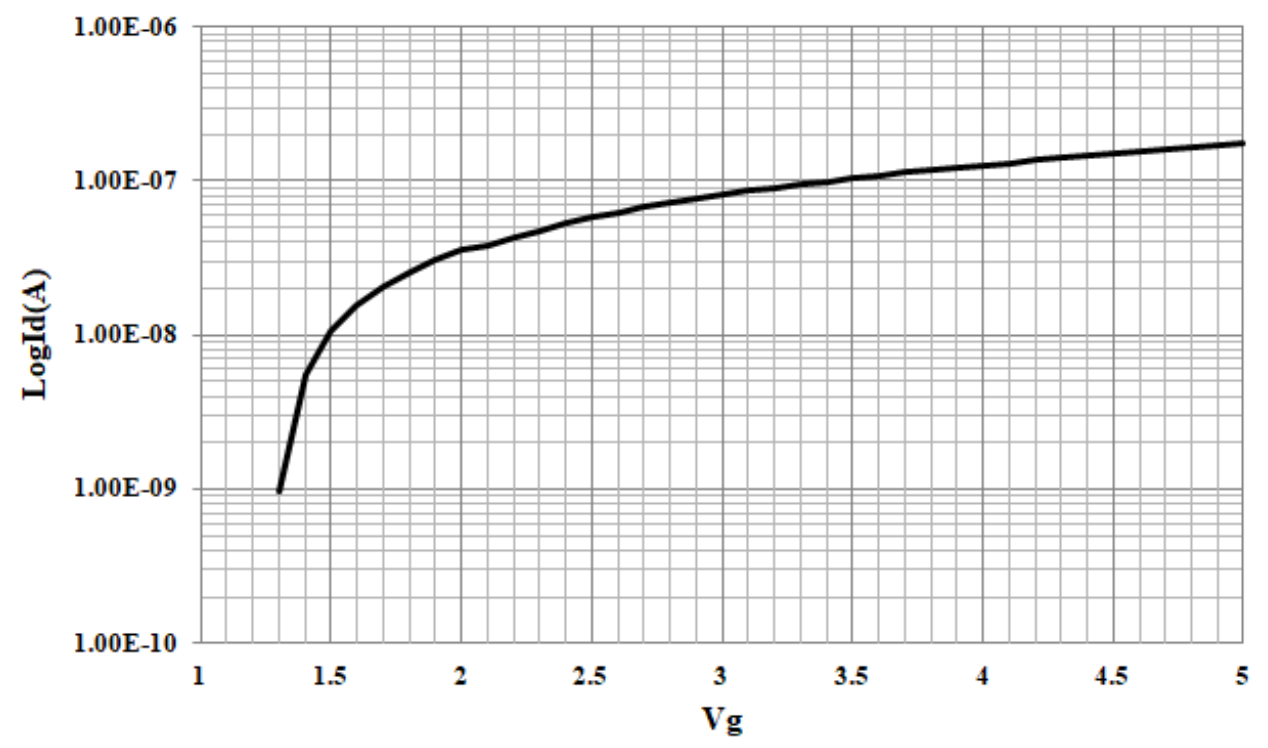

Figure 4. 13: Transfer characteristics of a SnSe n-FET.

Some of the 2D layered structures including black phosphorus (BP), $\mathrm{WSe}_{2}$, $\mathrm{WS}_{2} / \mathrm{SnS}$ heterostructure, etc. have previously been reported to exhibit ambipolar behavior. Recently SnS on top of black phosphorus also showed good ambipolar characteristics. 
Although SnSe has been reported to be an intrinsic p-type semiconductor, the ALD synthesized FET devices in our case showed both $\mathrm{p}$ - and $\mathrm{n}$-type behavior. The $\mathrm{I}_{\mathrm{d}}-\mathrm{V}_{\mathrm{g}}$ transfer characteristic of the n-FET is shown in Fig. 4.13. The calculated electron mobility of the device is found to be $\sim 0.7 \mathrm{~cm}^{2} /(\mathrm{V} . \mathrm{s})$ while the $\mathrm{I}_{\text {on }} / \mathrm{I}_{\text {off }}$ ratio is $\sim 10^{2}$. The $\mathrm{n}$-type behavior of SnSe thin film is unknown and needs further investigation. However good ambipolar characteristics of SnSe will enable the application of these devices in modern CMOS process without the need for doping methods.

\begin{tabular}{|c|c|c|}
\hline $\begin{array}{l}\text { Synthesis } \\
\text { Technique }\end{array}$ & $\begin{array}{l}\text { Mobility (p type) } \\
\mathrm{cm}^{2} / \mathrm{V} . \mathrm{s}\end{array}$ & Source \\
\hline $\begin{array}{l}\text { Chemical Vapor } \\
\text { Deposition }\end{array}$ & $\sim 2.6$ & Ref. 67 \\
\hline $\begin{array}{l}\text { Exfoliation from } \\
\text { bulk single crystals }\end{array}$ & $\sim 2.7$ & Ref. 57 \\
\hline $\begin{array}{l}\text { Vapor Transport } \\
\text { Deposition }\end{array}$ & $\sim 1.5$ & Ref. 7 \\
\hline $\begin{array}{l}\text { Thermal } \\
\text { Evaporation }\end{array}$ & $\sim 6.1$ & Ref. 29 \\
\hline $\begin{array}{l}\text { Atomic Layer } \\
\text { Deposition }\end{array}$ & $\sim 10$ & This work using ALD [Ref. 52] \\
\hline
\end{tabular}

Table 2: Literature review of SnSe hole mobility reported by various techniques.

P-type hole mobility of the as-grown SnSe thin film is amongst the highest that have been recently reported by other groups. CVD grown mobility was found $2.6 \mathrm{~cm}^{2} / \mathrm{V}$.s in 2018, exfoliated SnSe had a mobility of around $2.7 \mathrm{~cm}^{2} / \mathrm{V} . \mathrm{s}$ as reported in 2017 [67, 57]. 
Reported mobilities for vapor deposition technique and thermal evaporation were 1.5 $\mathrm{cm}^{2} /$ V.s and $6.1 \mathrm{~cm}^{2} /$ V.s respectively $[7,29]$. In the case of ALD grown SnSe we have found $10 \mathrm{~cm}^{2} /$ V.s [52]. A complete list of mobilities found by various techniques is illustrated in Table 2. 


\section{CHAPTER 5 \\ Conclusion and future work}

\subsection{Conclusion}

Beyond graphene, other novel 2D materials such as metal chalcogenides, black phosphorus, hBN etc. have also gained interest recently due to their unique properties and applications that emerge upon 2D carrier confinement. In this research one of the most promising two-dimensional layered metal chalcogenides from group IV-VI, tin selenide (SnSe) was investigated due to its potential applications in optoelectronics, photovoltaics and other areas. With its remarkable structural and electronic properties, SnSe is the material of interest for next generation of opto- and microelectronic devices. In order to realize these applications, techniques need to be developed to produce uniform films of this material over large areas. Hence, the objective of this research was develop such a growth technique and show that the films produced were comparable to those produced by exfoliation, which has been considered to be the gold standard of 2D materials.

Various synthesis methods have been reported in literature for growing the SnSe thin films, however none of these methods can produce uniform films over large areas. Hence, ALD was investigated for growth of SnSe for the goal of producing uniform, smooth and continuous films over the lager areas. As a result of this investigation, this goal was achieved, demonstrating that self-terminating reaction nature of the process is one of the greatest benefits of the ALD growth for precise controllability of the film.

The electrical characterization of the ALD synthesized SnSe films using fabricated back-gated FETs showed p-type transistor behavior with moderate hole mobility and 
yielded high $\mathrm{I}_{\text {on }} / \mathrm{I}_{\text {off }}$ ratios, demonstrating suitability for advanced semiconductor devices. Raman, XRD and XPS characterizations confirmed crystalline and orthorhombic phase of the as-grown SnSe films consistent with the characteristics reported in literature for several other growth techniques. Magnetic properties showed paramagnetic behavior for the thin film SnSe. The optical band gap energy of $1.74 \mathrm{eV}$ as characterized from the SnSe film makes it a promising material for optoelectronic applications. All these properties show that ALD grown films possess the necessary characteristics for their commercial applications.

\subsection{Future work}

Several hundred transistors were fabricated and tested on numerous SnSe substrates (more than 10 substrates) with different thicknesses ranging between $5 \mathrm{~nm}$ and $10 \mathrm{~nm}$. The mobility of 2D layered materials has a non-monotonic relation with layer thickness. Due to improved screening of charge impurities at the first few layers, mobility initially increases with the number of layers. However it tends to decrease in thicker samples due to increased interlayer resistance [68]. A detail study of the layer dependent mobility on SnSe thin films is necessary and this work can be extended by optimizing the number of layers in order to maximizing the hole mobility using the ALD growth sequence.

We can fabricate top gated monolithic transistors, where better gate control can be achieved by depositing a thin layer of gate oxide. This will yield higher drain saturation current and larger $I_{o n} / I_{\text {off }}$ ratio as compared to the back-gated transistor for practical switching devices. A prototype of the top-gated SnSe FET device is shown in Fig. 5.1. 


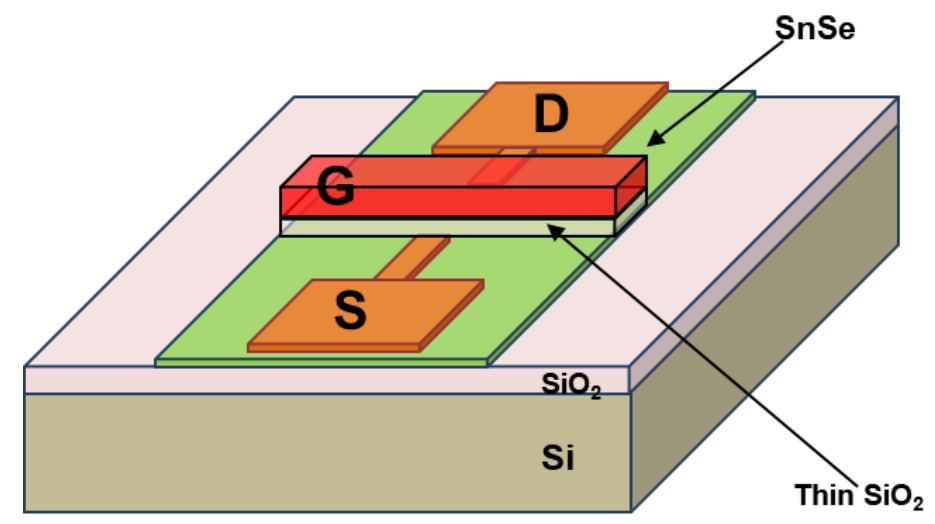

Figure 5 1: Schematic diagram of a Top-gated SnSe FET transistor.

Since the synthesized SnSe film has a direct bandgap, one could design and fabricate optical devices using these thin films. Furthermore, the optical devices can be integrated with the top gated FETs in monolithic transistors for next generation of novel devices. 


\section{References}

[1] M. Velicky, P. S. Toth, "From two-dimensional materials to their heterostructures: An electrochemist's perspective", Applied Materials Today 8 (2017) 68-103.

[2] G. Giovannetti, P.A. Khomyakov, G. Brocks, P.J. Kelly, J. V. D. Brink, "SubstrateInduced Band Gap in Graphene on Hexagonal Boron Nitride: Ab Initio Density Functional Calculations", Phys. Rev. B: Condens. Matter Mater. Phys. 2007, 76, 073103.

[3] A.C. Ferrari, F. Bonaccorso, V. Falko, K.S. Novoselov, S. Roche, P. Bøggild, S. Borini, F. Koppens, V. Palermo, N. Pugno et al. "Science and technology roadmap for graphene, related two-dimensional crystals, and hybrid systems", Nanoscale 2014, 7, $4598-4810$.

[4] K. Shavanova, Y. Bakakina, I. Burkova, I. Shtepliuk, R. Viter, A. Ubelis, V. Beni, N. Starodub, R. Yakimova and V. Khranovskyy, "Application of 2D Non-Graphene Materials and 2D Oxide Nanostructures for Biosensing Technology", Sensors 2016, 16, 223; doi:10.3390/s16020223.

[5] J.C. Slonczewski, P.R. Weiss, Band structure of graphite, Phys. Rev. 109(1958) $272-279$.

[6] L.F. Mattheiss, Band structures of transition-metal-dichalcogenide layercompounds, Phys. Rev. B 8 (1973) 3719-3740.

[7] S. Zhao, H. Wang, Y. Zhou, L. Liao, Y. Jiang, X. Yang, G. Chen, M. Lin,Y. Wang, H. Peng, and Z. Liu, "Controlled synthesis of singlecrystal SnSe nanoplates", Nano Res. (2015) 8: 288.

[8] F. Xia, H. Wang, D. Xiao, M. Dubey and A. Ramasubramaniam, "Twodimensional material nanophotonics", Nature Photonics volume 8, pages 899-907 (2014).

[9] P. A. Lee ed., "Optical and Electrical Properties, Physics and Chemistry of Materials with Layered Structures", vol. 4 (D. Riedel Pub. Co., Dordrecht, 1976).

[10] V. Grasso ed., "Electronic Structure and Electronic Transitions in Layered Materials, Physics and Chemistry of Materials with Low- Dimensional Structures", series A, (D. Riedel Pub. Co., Dordrecht, 1986).

[11] N. K. Reddy, M. Devika, E. S. R. Gopal, "Review on Tin (II) Sulfide (SnS) Material: Synthesis, Properties, and Applications", Crit. Rev. Solid State Mater. Sci. 2015, 40, 359.

[12] V. R. M. Reddy, S. Gedi, B. Pejjai, C. Park,"Perspectives on SnSe-based thin film solar cells: a comprehensive review", J.Mater. Sci.: Mater. Electron. 2016, 27, 5491.

[13] J. P. Heremans, V. Jovovic, E. S. Toberer, A. Saramat, K. Kurosaki, A. Charoenphakdee, S. Yamanaka, and G. J. Snyder,"Enhancement of Thermoelectric Efficiency in PbTe by Distortion of the Electronic Density of States " Science 321, 554 (2008).

[14] Q. Zhang, H. Wang, W. Liu, et al,"Enhancement of thermoelectric figure-of-merit by resonant states of aluminium doping in lead selenide" Energy Environ. Sci. 5, 5246 (2012). 
[15] D. Wu, L. Zhao, S. Hao, et al, "Origin of the High Performance in GeTe-Based Thermoelectric Materials upon $\mathrm{Bi}_{2} \mathrm{Te}_{3}$ Doping"J. Am. Chem. Soc. 136, 11412 (2014).

[16] G. Tan, L. Zhao, F. Shi, J. W. Doak, S. Lo, H. Sun, C. Wolverton, V. P. Dravid, C. Uher, and M. G. Kanatzidis, "High Thermoelectric Performance of p-Type SnTe via a Synergistic Band Engineering and Nanostructuring Approach" J. Am. Chem. Soc. 136, 7006 (2014).

[17] L. D. Zhao, S. H. Lo, Y. Zhang, H. Sun, G. Tan, C Uher, C. Wolverton, V. P. Dravid \& M. G. Kanatzidis, "Ultralow thermal conductivity and high thermoelectric figure of merit in SnSe crystals", Nature volume 508, pages 373-377 (17 April 2014).

[18] Cheng-Lung Chen, Heng Wang, Yang-Yuan Chen, Tristan Daya and G. Jeffrey Snyder, "Thermoelectric properties of p-type polycrystalline SnSe doped with Ag", J. Mater. Chem. A, 2014, 2, 11171-11176.

[19] N. Kh. Abriksov, V. F. Bankina, L. V. Poretskaya, L. E. Shelimova and E.V. Skudnova, "Semiconducting II-VI, IV-VI, and V-VI Compounds", Plenum Press, New York, 1969, pp. 74-82.

[20] L.-D. Zhao, G. Tan, S. Hao, J. He, Y. Pei, H. Chi, H. Wang, S. Gong, H. Xu, V. P. Dravid, C. Uher, G. J. Snyder, C. Wolverton, and M. G. Kanatzidis, "Ultrahigh power factor and thermoelectric performance in hole-doped single-crystal SnSe", Science 351, 141 (2016).

[21] D. Chun, R. M. Walser, R. W., Bene, T. H. Courtney, "Polarity dependent memory switching in devices with SnSe and SnSe 2 crystals",Appl. Phys. Lett. 1974, 24, 479481.

[22] A. Agarwal, M. N. Vashi, D. Lakshminarayana, N. M. Batra, "Electrical resistivity anisotropy in layered p-SnSe single crystals” J. Mater. Sci- Mater. El. 2000, 11, 67-71.

[23] N. D. Boscher, C. J. Carmalt, R. G. Palgrave, I. P. Parkin, "Atmospheric pressure chemical vapor deposition of $\mathrm{SnSe}$ and $\mathrm{SnSe}_{2}$ thin films on glass", Thin Solid Films 2008, 516, 4750-4757.

[24] C. K. Sumesh, M. Patel, K. D. Patel, G. K Solanki, V. M. Pathak, R. Srivastav, "Low temperature electrical transport properties in p-SnSe single crystals", Eur. Phys. J-Appl.Phys. 2011, 53, 10302.

[25] M. Z. Xue, J. Yao, S. C. Cheng, Z. W. Fu, "Lithium electrochemistry of a novel SnSe thin-film anode", J. Electrochem. Soc. 2006, 153, A270- A274.

[26] R.Y. Wang, M.A. Caldwell, R.G.D. Jeyasingh, S. Aloni, R.M. Shelby, H-SP. Wong, D.J. Milliron, "Electronic and optical switching of solution-phase deposited $\mathrm{SnSe}_{2}$ phase change memory material", J Appl Phys 109:113506 (2011).

[27] H. Song and J. Lü, "Density functional theory study of inter-layer coupling in bulk tin selenide", Chemical Physics Letters 695 (2018) 200-204.

[28] L. Das, A. Guleria and S. Adhikari, "Aqueous phase one-pot green synthesis of $\mathrm{SnSe}$ nanosheets in a protein matrix: negligible cytotoxicity and room temperature emission in the visible region", RSC Adv., 2015, 5, 61390-61397.

[29] R. Indirajith, T.P. Srinivasan, K. Ramamurthi, R. Gopalakrishnan, "Synthesis, deposition and characterization of tin selenide thin films by thermal evaporation technique", Current Applied Physics 10 (2010) 1402-1406.

[30] M. Bicer, I. Sisman, "Electrodeposition and growth mechanism of SnSe thin films". Appl. Surf. Sci. 257, 2944-2949 (2011). 
[31] R. Mariappan, V. Ponnuswamy, M. Ragavendar, "Effects of substrate temperature on the properties of CdSnSe thin films deposited by nebulizer spray pyrolysis technique", Mater. Sci. Semicond. Process. 15, 199-205 (2012).

[32] S. Anwar, S. Gowthamaraju, B.K. Mishra, S.K. Singh, S. Anwar, "Spray pyrolysis deposited tin selenide thin films for thermoelectric applications", Mater. Chem. Phys. 153, 236-242 (2015).

[33] D. Martinez-Escobar, M. Ramchandaran, A. Sanchez-Juarez, J.S.N. Rios, "Optical and electrical properties of $\mathrm{SnSe}_{2}$ and $\mathrm{SnSe}$ thin films prepared by spray pyrolysis", Thin Solid Films 535, 390-393 (2013).

[34] J.P. Singh, "Transport and optical properties of hot wall grown tin selenide films", J. Mater. Sci.: Mater. Electron. 2, 105-108 (1991).

[35] D.P. Padiyan, A. Marikani, K.R. Murali, "Electrical and photoelectrical properties of vacuum deposited SnSe thin films", Cryst. Res. Technol. 35, 949-957 (2000).

[36] R. Teghil, A. Santagata, V. Marotta, S. Orlando, G. Pizzela, A. Giordini- Guidoni, A. Mele, "Characterization of the plasma plume and of thin film epitaxially produced during laser ablation of SnSe", Appl. Surf. Sci. 90, 505-514 (1995).

[37] F.A. Fernandes, M.G. Sausa, P.M.P. Salome, J.P. Leitao, A.F. da Cunha, "Thermodynamic pathway for the formation of $\mathrm{SnSe}$ and $\mathrm{SnSe}_{2}$ polycrystalline thin films by selenization of metal precursors", Cryst. Eng. Commun. 15, 10278-10286 (2013).

[38] N.D. Boscher, C.J. Carmalt, R.G. Palgrave, I.P. Parkin, "Atmospheric pressure chemical vapour deposition of $\mathrm{SnSe}$ and $\mathrm{SnSe}_{2}$ thin films on glass", Thin Solid Films $516,4750-4757$ (2008).

[39] Z. Zainal, N. Sarvanan, K. Anuar, M.Z. Hussein, W.M.M. Yunus, "Chemical bath deposition of tin selenide thin films", Mater. Sci. Eng. B 107, 181-185 (2004).

[40] R. W. Johnson, A. Hultqvist and S. F. Bent, "A brief review of atomic layer deposition: from fundamentals to applications", Materials Today, Volume 17, Issue 5, June 2014, Pages 236-246.

[41] C. S. Hwang,"Atomic Layer Deposition of Nanostructured Materials", (Eds: N. Pinna, M. Knez), Wiley-VCH, 2011, 161.

[42] S. M. George,"Atomic Layer Deposition: An Overview", Chem. Rev. 2010, 110, 111.

[43] M. Knez, K. Niesch, L. Niinisto, "Synthesis and Surface Engineering of Complex Nanostructures by Atomic Layer Deposition", Adv. Mater. 2007, 19, 3425.

[44] M. Leskelä, M. Ritala,"Atomic Layer Deposition Chemistry: Recent Developments and Future Challenges", Angew. Chem., Int. Ed. 2003, 42, 5548.

[45] N. Pinna, M. Knez, "Atomic Layer Deposition of Nanostructured Materials", Wiley-VCH, 2011.

[46] R. L. Puurunen, "Surface chemistry of atomic layer deposition: A case study for the trimethylaluminum/water process", J. Appl. Phys. 2005, 97, 121301.

[47] M. Ritala and M. Leskela, in: H. S. Nalwa, (Ed), "Handbook of Thin Film Materials", Academic Press, San Diego, 2001, Vol. 1, Chapter 2, p 103.

[48] O. Sneh, R.B. Clark-Phelps, A.R. Londergan, J.L. Winkler and T.E. Seidel, "Thin film atomic layer deposition equipment for semiconductor processing", Thin Solid Films, 402/1-2 (2002) 248. 
[49] J. E. Green, in: R. F. Bunshah (Ed), "Handbook of Deposition Technologies for Films and Coatings", 2nd edition, Noyes Publications, Park Ridge, NJ, 1994, Chap. 13, p.681.

[50] S.S. Dana, M. Liehr, M. Anderle and G. W. Rubloff, "Kinetics of nucleation and growth of $\mathrm{Si}$ on $\mathrm{SiO}_{2}$ in very low pressure $\mathrm{SiH} 4$ chemical vapor deposition”, Appl. Phys. Lett. 61 (1992) 3035.

[51] O. Bethge, S. Abermann, C. Henkel and E. Bertagnolli, "Low temperature atomic layer deposition of high-k dielectric stacks for scaled metal-oxide-semiconductor devices", Thin Solid Films, 2009. 517(18): p. 5543-5547.

[52] S. Afrin, N. Kuperman and R. Solanki, "Atomic layer deposition of 2-dimensional, semiconducting SnSe thin films", 2018 IEEE 13th Nanotechnology Materials and Devices Conference (NMDC), 2018 IEEE.

[53] H. R. Chandrasekhar, R. G. Humphreys, U. Zwick, and M. Cardona, "Infrared and Raman spectra of the IV-VI compound SnS and SnSe". Phys. Rev. B 15, 2177.

[54] C. Gurnani, S. L. Hawken, A. L. Hector, R. Huang, M. Jura, W. Levason, J. Perkins, G. Reid and G. B. G. Stenning, "Tin(IV) chalcogenoether complexes as single source precursors for the chemical vapour deposition of $\mathrm{SnE}_{2}$ and $\mathrm{SnE}(\mathrm{E}=\mathrm{S}, \mathrm{Se})$ thin films". Dalton Trans., 2018, 47, 2628-2637.

[55] M. N. Ashiq, S. Irshad, M. F. Ehsan, S. Rehman, S. Farooq, M. N. Haqa and A. Ziab, "Visible-light active tin selenide nanostructures: synthesis, characterization and photocatalytic activity". New J. Chem., 2017, 41, 14689-14695.

[56] W. Zhang, Z. Yang, J. Liu, L. Zhang, Z. Hui, W. Yu, Y. Qian, L. Chen, X. Liu,"Room temperature growth of nanocrystalline tin (II) selenide from aqueous solution”. Volume 217, Issues 1-2, 11 July 2000.

[57] S. H. Cho, K. Cho, N. W. Park, S. Park, J. H. Koh, and S. K. Lee, "Multi-layer SnSe nanoflake field effect transistors with low-resistance Au Ohmic contacts", Nanoscale Res. Lett. 12, 273 (2017).

[58] Ferreira, Carlos Sérgio, Santos, Pãmyla Layene, Bonacin, Juliano Alves, Passos, Raimundo Ribeiro, \& Pocrifka, Leandro Aparecido. (2015). "Rice Husk Reuse in the Preparation of $\mathrm{SnO}_{2} / \mathrm{SiO}_{2}$ Nanocomposite", Materials Research, 18(3), 639-643.

[59] G. Shi and E. Kioupakis, "Anisotropic spin transport and strong visible-light absorbance in few-layer SnSe and GeSe", Nano Lett. 2015, 15, 6926-6931.

[60] M. A. Franzman, C. W. Schlenker, M. E. Thompson, R. L. Brutche, "SolutionPhase Synthesis of SnSe Nanocrystals for Use in Solar Cells", J. Am. Chem. Soc. 2010, $132,4060$.

[61] N. kumar, U. Parihar, R. Kumar, K. J. Patel, C. J. Panchal and N. Padha, "Effect of Film Thickness on Optical Properties of Tin Selenide Thin Films Prepared by Thermal Evaporation for Photovoltaic Applications", American Journal of Materials Science 2012, 2(1): 41-45.

[62] P. K. Nair, E. Barrios-Salgado, M. T. S. Nair, "Cubic-structured tin selenide thin film as a novel solar cell absorber", Phys. Status Solidi A 2016, 213, 2229.

[63] E. Barrios-Salgado, L. A. Rodríguez-Guadarrama, A. R. Garcia-Angelmo, J. C. Álvarez, M. T. S. Nair, P. K. Nair, "Large cubic tin sulfide-tin selenide thin film stacks for energy conversion", Thin Solid Films 2016, 615, 415. 
[64] S. Liu, X. Guo, M. Li, W. Zhang, X. Liu, and C. Li, "Solution-Phase Synthesis and Characterization of Single-Crystalline SnSe Nanowires", Angew. Chem. Int. Ed., DOI: 10.1002/anie.201105614.

[65] W. Shi, M. Gao, J. Wei, J. Gao, C. Fan, E. Ashalley, H. Li, and Z. Wang, "Tin Selenide (SnSe): Growth, Properties, and Applications", Adv. Sci. 2018, 5, 1700602.

[66] D. De, J. Manongdo, S. See, V. Zhang, A. Guloy and H. Peng, "High on/off ratio field effect transistors based on exfoliated crystalline $\mathrm{SnS}_{2}$ nano-membranes", Nanotechnology. 2013 Jan 18; 24(2):025202.

[67] S. Liu, N. Sun, M. Liu, S. Sucharitakul and X. P. A. Gao, "Nanostructured SnSe: Synthesis, doping, and thermoelectric properties", Journal of Applied Physics 123, 115109 (2018).

[68] S. Das, H.-Y. Chen, A. V. Penumatcha, J. Appenzeller, "High Performance Multilayer $\mathrm{MoS}_{2}$ Transistors with Scandium Contacts", Nano Letters 2012, 13, 100105. 